Arkivoc

Free to Authors and Readers
A Platinum Open Access Journal for Organic Chemistry
Paper

Arkivoc 2022, part iv, 6-23

\title{
Approaches to calix[3]indoles from activated indole carboxylic acids
}

\author{
Bambang Purwono, Naresh Kumar, and David StC. Black* \\ School of Chemistry, UNSW Sydney, Sydney, NSW 2052, Australia \\ Email:d.black@unsw.edu.au
}

Received 09-09-2021

Accepted 10-05-2021

Published on line 10-07-2021

\section{Abstract}

Acid-catalyzed reactions of 3-substituted 4,6-dimethoxyindole-7- and -2-carboxylic acids with aryl aldehydes generate unsymmetrically-oriented calix[3]indoles, with one 2,2'-linkage, one 2,7'-linkage and one 7,7'linkage, in a wide range of yields. This behavior contrasts with similar reactions of the parent indoles, which more commonly yield the symmetrically-oriented calix[3]indoles, with three 2,7'-linkages. The starting material carboxylic acids were prepared via the hydrolysis of trifluoroacetyl and trichloroacetyl substituents formed by acylation of the parent 3-substituted-4,6-dimethoxyindoles.<smiles>[R]c1c[nH]c2c(C(=O)O)c(OC)cc(OC)c12</smiles>

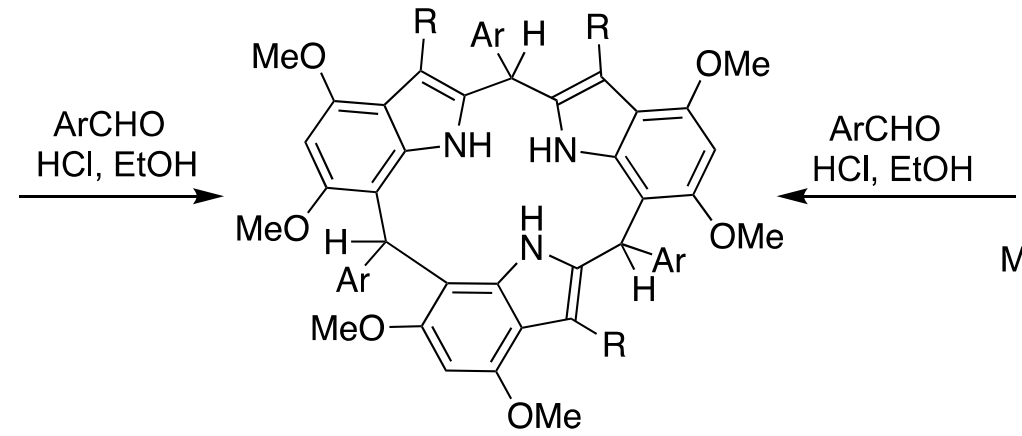<smiles>[R]c1c(C(=O)O)[nH]c2cc(OC)cc(OC)c12</smiles>

Keywords: Indoles, carboxylic acids, calix[3]indoles, acid-catalysis, macrocycles 


\section{Introduction}

We have previously reported the synthesis of calix[3]indoles from indole precursors activated by the presence of methoxy groups at $\mathrm{C} 4$ and $\mathrm{C} 6 .{ }^{1-6}$ Two direct methods have been used. The first involves the combination of a 3-substituted-4,6-dimethoxyindole with an aryl carbaldehyde under acidic conditions. The second requires the treatment of either 2- or 7-hydroxyalkyl-3-substituted-4,6-dimethoxyindoles with acid. Two different structural isomers of calix[3]indoles can be formed, depending on the reaction conditions. One has three $2,7^{\prime}-$ linkages (the so-called symmetrically-oriented isomer) and the other has one 2,2'-linkage, one 2,7'-linkage and one $7,7^{\prime}$-linkage (the so-called unsymmetrically-oriented isomer) (Scheme 1 ). The symmetrically-oriented isomer generally takes up a flattened partial cone conformation, but under certain circumstances a cone conformation can be achieved by the introduction of suitable hydrogen bonding. ${ }^{4,5}$ In addition, application of the second method can give rise to the formation of calix[4]indoles containing four 2,7'-linkages. There are four possible structural isomers of calix[4]indoles. A third method using a stepwise set of reactions can be applied to the synthesis of all possible calixindole isomers. In this paper we consider only the first two direct methods and the application of suitable indole carboxylic acids to the formation of calix[3]indoles, with concomitant decarboxylation. In general, we have found that in reactions of activated indoles with aryl aldehydes, under acidic reaction conditions involving phosphoryl chloride, the symmetrically-oriented isomer is selectively formed in rapid reactions with strongly electrophilic aldehydes, whereas the unsymmetricallyoriented isomer is preferred in slower reactions with weakly electrophilic aldehydes. We therefore decided to investigate reactions of the related less reactive indole carboxylic acids with aryl aldehydes, in the desire to achieve a simple, direct formation of unsymmetrically-oriented calix[3]indoles as a result of slower reactions.
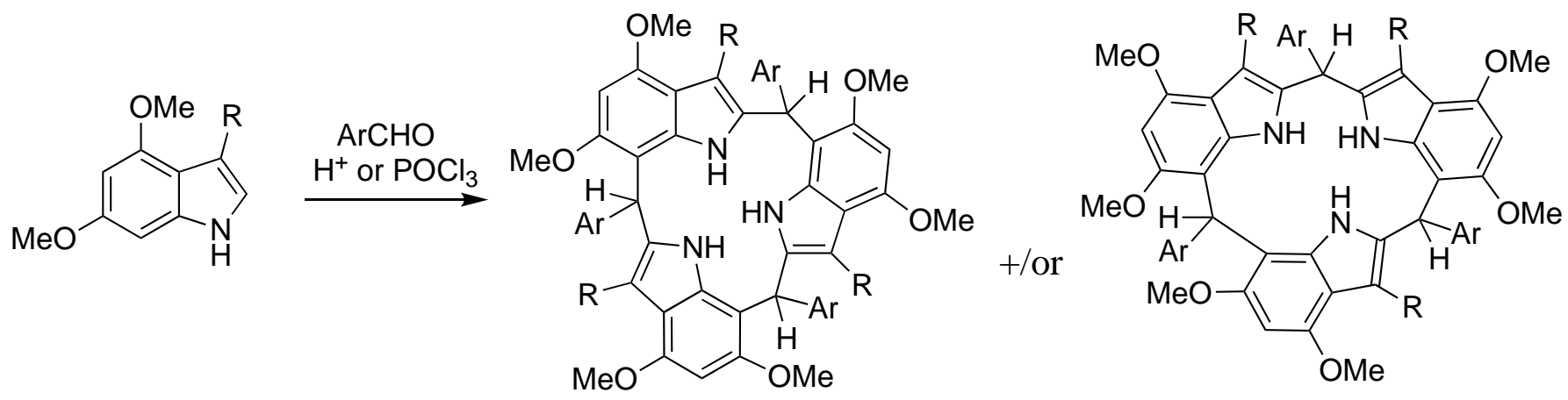<smiles>[R]c1c[nH]c2c(C([Y])O)c(OC)cc(OC)c12</smiles><smiles>[R]c1c(C([X])O)[nH]c2cc(OC)cc(OC)c12</smiles>

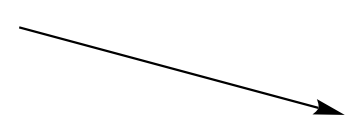

$\mathrm{H}^{+}$

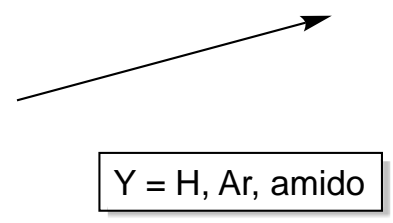

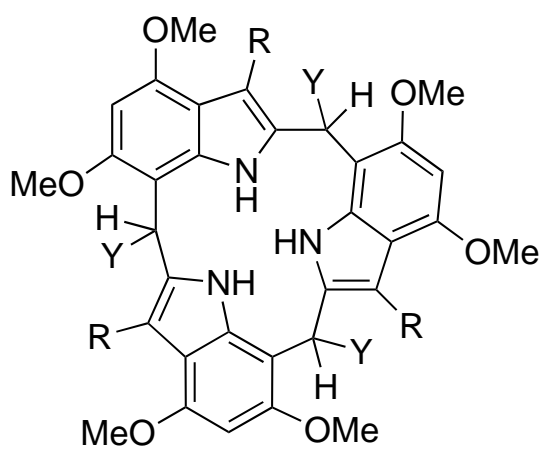

Scheme 1. Possible formation of calix[3]indoles from activated indole derivatives. 


\section{Results and Discussion}

\section{Preparation of the starting indole carboxylic acids}

The chosen starting materials comprised examples of indole-7- and 2-carboxylic acids, indole-2,7-dicarboxylic acids, and 2-hydroxyalkylindole-7- and 2-carboxylic acids. These new compounds were prepared by routine reactions which made use of the activation bestowed on the indole nucleus by the methoxy groups. Treatment of the 3-arylindole 1 with trifluoroacetic anhydride gave the 7-trifluoracetyl indole 3 and the 2,7ditrifluoroacetylindole 7, but not the 2-trifluoroacetylindole 5 (Scheme 2). Similar reaction of the 3methylindole 2 gave the 2-trifluoroacetylindole 6 and the 2,7-ditrifluoroacetylindole 8, but not the 7trifluoroacetylindole $\mathbf{4}$. Treatment of indole $\mathbf{1}$ with trichloroacetyl chloride gave the 7-trichloroacetylindole $\mathbf{9}$ and the 2-trichloroacetylindole 11, but not the 2,7-ditrichloroacetylindole 13. Similar reaction of indole 2 gave the 2-trichloroacetylindole 12 and the 2,7-ditrichloroacetylindole 14, but not the 7-trichloroacetylindole 10 (Scheme 2). Yields were generally low, but were not optimized because it was important to maintain the same conditions over the range of reactions for purposes of comparison.<smiles>[R]c1c[nH]c2cc(OC)cc(OC)c12</smiles>
$1 \mathrm{R}=4-\mathrm{ClC}_{6} \mathrm{H}_{4}$
$2 \mathrm{R}=\mathrm{Me}$
$3 \mathrm{R}=4-\mathrm{ClC}_{6} \mathrm{H}_{4}(35 \%)$
$4 \mathrm{R}=\mathrm{Me}(0 \%)$
$5 \mathrm{R}=4-\mathrm{ClC}_{6} \mathrm{H}_{4}(0 \%)$
$6 \mathrm{R}=\mathrm{Me}(20 \%)$
$7 \mathrm{R}=4-\mathrm{ClC}_{6} \mathrm{H}_{4}(25 \%)$
$8 \mathrm{R}=\mathrm{Me}(5 \%)$<smiles>[R]c1c[nH]c2cc(OC)cc(OC)c12</smiles><smiles>[R]c1c[nH]c2c(C(=O)C(Cl)(Cl)C(Cl)(Cl)Cl)c(OC)cc(OC)c12</smiles><smiles>[R]c1c(C(=O)C(F)(F)Cl)[nH]c2cc(OC)cc(OC)c12</smiles>

$1 \mathrm{R}=4-\mathrm{ClC}_{6} \mathrm{H}_{4}$

$2 \mathrm{R}=\mathrm{Me}$

$9 \mathrm{R}=4-\mathrm{ClC}_{6} \mathrm{H}_{4}(66 \%)$

$10 \mathrm{R}=\mathrm{Me}(0 \%)$

$11 \mathrm{R}=4-\mathrm{ClC}_{6} \mathrm{H}_{4}(17 \%)$

$12 \mathrm{R}=\mathrm{Me}(21 \%)$<smiles>[R]c1c(C(=O)C(Cl)(Cl)Cl)[nH]c2c(C(=O)C(Cl)(Cl)Cl)c(OC)cc(OC)c12</smiles>

Scheme 2. Reaction of indoles $\mathbf{1}$ and $\mathbf{2}$ with trifluoroacetic anhydride and trichloroacetyl chloride.

The 3-arylindole-7-carboxylic acid $\mathbf{1 5}$ was obtained by base hydrolysis of either the trifluoroacetylindole $\mathbf{3}$ or the trichloroacetylindole $\mathbf{9}$ (Scheme 3). The 3-arylindole-2-carboxylic acid $\mathbf{1 6}$ was similarly derived from the trichloroacetylindole 11, while the 3-methylindole-2-carboxylic acid $\mathbf{1 7}$ was derived from the trifluoroacetylindole $\mathbf{6}$. Base hydrolysis of the ditrifluoroacetylindole $\mathbf{8}$ gave the 3-methylindole-2,7dicarboxylic acid 18 (Scheme 3). Yields were generally high. The choice of sodium or potassium hydroxide relates to the solubility of the indoles. Conditions for the hydrolysis of trichloroacetyl compounds have been reported to use sodium hydroxide, so these were followed. ${ }^{7}$ 
<smiles>[R]c1c[nH]c2c(C(=O)C(F)(F)F)c(OC)cc(OC)c12</smiles>

$3 \mathrm{R}=4-\mathrm{ClC}_{6} \mathrm{H}_{4}$
$\mathrm{KOH}, \mathrm{EtOH}, \mathrm{H}_{2} \mathrm{O}$ reflux, $3 \mathrm{~h}$<smiles>[R]c1c[nH]c2c(C(=O)O)c(OC)cc(OC)c12</smiles>

$\mathrm{NaOH}, \mathrm{EtOH}, \mathrm{H}_{2} \mathrm{O}$ reflux, $1 \mathrm{~h}$

$84 \%$<smiles>[R]c1c[nH]c2c(C(=O)C(F)(Cl)C(F)(F)Cl)c(OC)cc(OC)c12</smiles>

$15 \mathrm{R}=4-\mathrm{ClC}_{6} \mathrm{H}_{4}$

$9 \mathrm{R}=4-\mathrm{ClC}_{6} \mathrm{H}_{4}$<smiles>[R]c1c(C(=O)C(F)(Cl)Cl)[nH]c2cc(OC)cc(OC)c12</smiles>

$11 \mathrm{R}=4-\mathrm{ClC}_{6} \mathrm{H}_{4}$<smiles>[R]c1c(C(=O)O)[nH]c2cc(OC)cc(OC)c12</smiles>

$16 \mathrm{R}=4-\mathrm{ClC}_{6} \mathrm{H}_{4}$ $17 \mathrm{R}=\mathrm{Me}$<smiles>[R]c1c(C(=O)C(F)(F)F)[nH]c2cc(OC)cc(OC)c12</smiles>

$6 \mathrm{R}=\mathrm{Me}$<smiles>[R]c1c(C(=O)C(F)(F)F)[nH]c2c(C(=O)C(F)(F)C(F)(F)F)c(OC)cc(OC)c12</smiles>

$8 \mathrm{R}=\mathrm{Me}$
$\mathrm{KOH}, \mathrm{EtOH}, \mathrm{H}_{2} \mathrm{O}$ reflux, $3 \mathrm{~h}$

$82 \%$<smiles>[R]c1c(C(=O)O)[nH]c2c(C(=O)O)c(OC)cc(OC)c12</smiles>

$18 \mathrm{R}=\mathrm{Me}$

Scheme 3. Formation of indole carboxylic and dicarboxylic acids 15-18.

The 2-hydroxymethylindole-7-carboxylic acid $\mathbf{2 1}$ was prepared in a sequence of reactions from the indole 9, involving formylation to give the 2-carbaldehyde 19, hydrolysis of the trichloroacetyl group to give the indole acid 20, followed by reduction of this compound with sodium borohydride to give the 2-hydroxymethylindole-7-carboxylic acid 21. In a corresponding reaction sequence, the indole $\mathbf{1 1}$ was formylated at $\mathrm{C7}$ to give the carbaldehyde 22: this was hydrolysed to the indole acid 23, which was reduced by sodium borohydride to give the 7-hydroxymethylindole-2-carboxylic acid $\mathbf{2 4}$ (Scheme 4). Neither hydroxy acids 21 nor 24 could be fully purified, so were submitted directly to further reactions (see later). 
<smiles>[R]c1c[nH]c2c(C(=O)C(Cl)(Cl)C(Cl)(Cl)Cl)c(OC)cc(OC)c12</smiles>

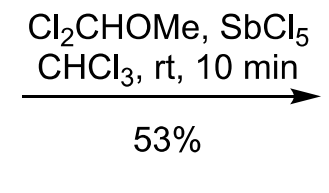

$9 \mathrm{R}=4-\mathrm{ClC}_{6} \mathrm{H}_{4}$<smiles>[R]c1c(CO)[nH]c2c(C(=O)O)c(OC)cc(OC)c12</smiles>

$21 \mathrm{R}=4-\mathrm{ClC}_{6} \mathrm{H}_{4}$<smiles>[R]C(=C=O)CCCCCCCCC(=O)C(=O)C(Cl)(Cl)Cl</smiles>

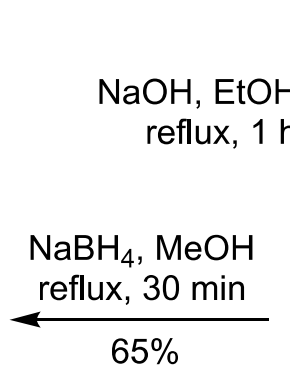

$65 \%$<smiles>[R]c1c(C(=O)C(Cl)(Cl)Cl)[nH]c2cc(OC)cc(OC)c12</smiles>

$11 \mathrm{R}=4-\mathrm{CIC}_{6} \mathrm{H}_{4}$

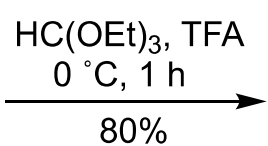

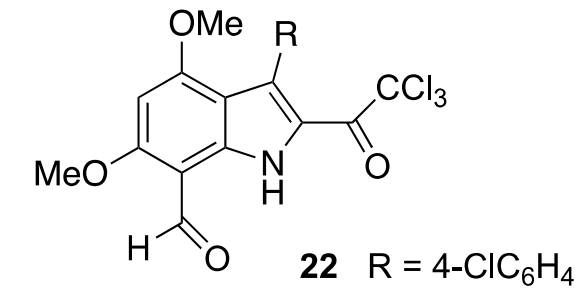

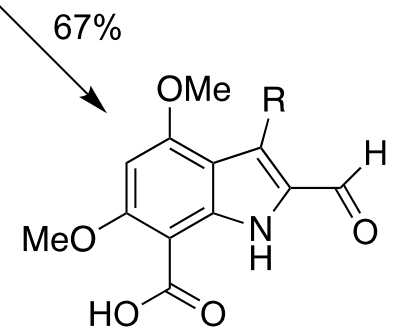

$20 \mathrm{R}=4-\mathrm{ClC}_{6} \mathrm{H}_{4}$
$24 \mathrm{R}=4-\mathrm{ClC}_{6} \mathrm{H}_{4}$<smiles>[R]c1c(C(=O)O)[nH]c2c(CO)c(OC)cc(OC)c12</smiles>

$87 \%$<smiles>[R]c1c(C(=O)O)[nH]c2c(CO)c(OC)cc(OC)c12</smiles>

Scheme 4. Formation of hydroxymethylindole carboxylic acids $\mathbf{2 1}$ and $\mathbf{2 4 .}$

\section{Reactions of indole carboxylic acid 15 with aryl aldehydes}

The indole-7-carboxylic acid $\mathbf{1 5}$ was heated under reflux with one equivalent of 4-chlorobenzaldehyde in ethanol containing concentrated hydrochloric acid and gave the unsymmetrically-oriented calix[3]indole 25 in $80 \%$ yield (Scheme 5). The structure of compound 25 was clear from NMR data, in particular with the application of ${ }^{1} \mathrm{H}-{ }^{13} \mathrm{C}$ and ${ }^{1} \mathrm{H}-{ }^{15} \mathrm{~N}$ correlations, which allowed a distinction between symmetrically-oriented and unsymmetrically-oriented structures. However, in this case, the calix[3]indole $\mathbf{2 5}$ was also synthesized using an unambiguous stepwise route. Indole 1 was reacted with 4-chloro- $N, N$-dimethylbenzamide and phosphoryl chloride to give a mixture of the 7-(4-chlorobenzoyl)indole 26 and the 2-(4-chlorobenzoyl)indole 27 in 62 and $13 \%$ yields, respectively. The 7-isomer 26 was reacted with 4-chlorobenzaldehyde in methanol containing concentrated hydrochloric acid to give the diindolylmethane $\mathbf{2 8}$ in $85 \%$ yield. Reaction of this compound with 
<smiles>[R]c1c[nH]c2c(C(=O)O)c(OC)cc(OC)c12</smiles>

$15 \mathrm{R}=4-\mathrm{ClC}_{6} \mathrm{H}_{4}$

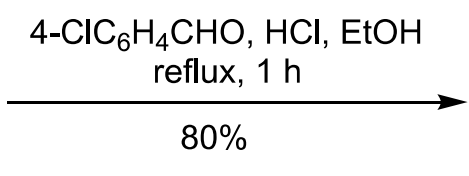<smiles>[R]c1c[nH]c2cc(OC)cc(OC)c12</smiles>

$1 \mathrm{R}=4-\mathrm{ClC}_{6} \mathrm{H}_{4}$

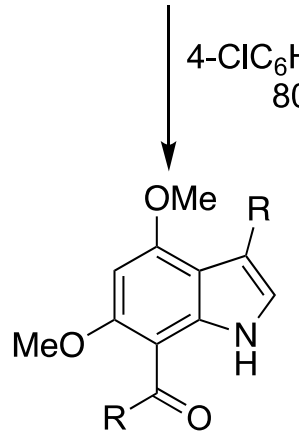

$26 \mathrm{R}=4-\mathrm{ClC}_{6} \mathrm{H}_{4}(62 \%)$<smiles>[R]C(=O)c1[nH]c2cc(OC)cc(OC)c2c1[R]</smiles>

$27 \mathrm{R}=4-\mathrm{ClC}_{6} \mathrm{H}_{4} \quad(13 \%)$

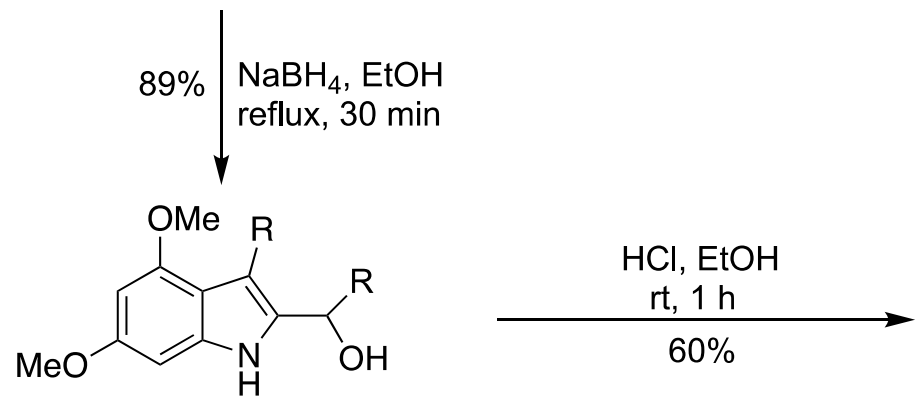

$30 \mathrm{R}=4-\mathrm{ClC}_{6} \mathrm{H}_{4}$

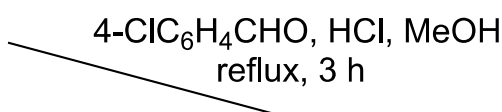

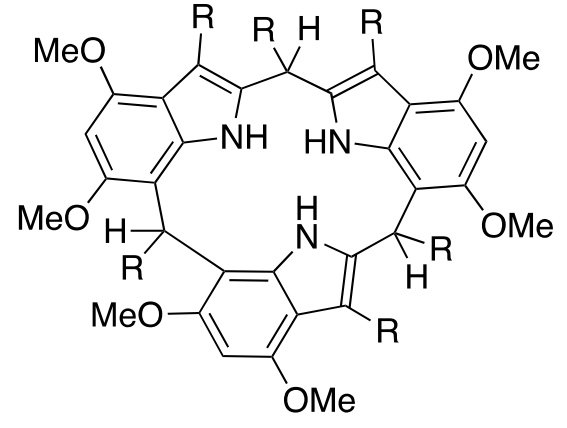

$25 \mathrm{R}=4-\mathrm{ClC}_{6} \mathrm{H}_{4}$

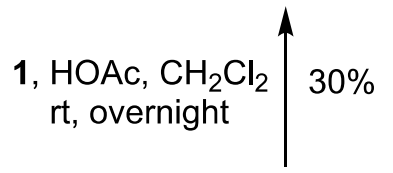<smiles>[R]c1c(C([2H])([2H])c2[nH]c3c(C([2H])([2H])O)c(OC)cc(OC)c3c2[R])[nH]c2c(C([2H])([2H])[2H])c(OC)cc(OC)c12</smiles>

$\mathrm{NaBH}_{4}, \mathrm{THF}$ reflux, 30 min $80 \%$
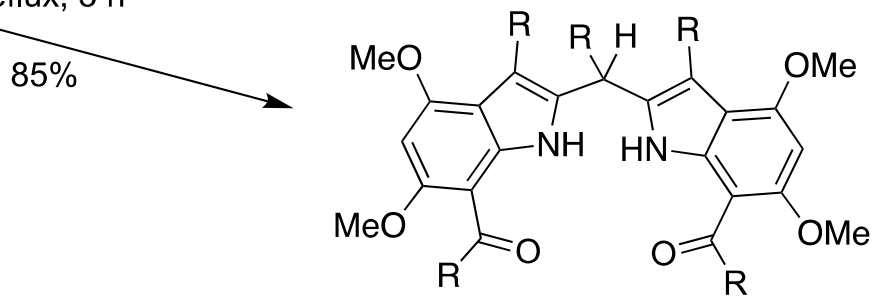

$28 \mathrm{R}=4-\mathrm{ClC}_{6} \mathrm{H}_{4}$<smiles>[R]c1c2[nH]c3c(c(OC)cc(OC)c13)C([R])([2H])c1c(OC)cc(OC)c3c([R])c([nH]c13)C([2H])([2H])c1c(OC)cc(OC)c3c([R])c([nH]c13)C2([2H])[2H]</smiles>

$31 \mathrm{R}=4-\mathrm{ClC}_{6} \mathrm{H}_{4}$

Scheme 5. Formation of calix[3]indoles $\mathbf{2 5}$ and $\mathbf{3 1 .}$ 
sodium borohydride gave the dialcohol 29, which was without purification directly combined with one equivalent of indole $\mathbf{1}$ in dichloromethane containing acetic acid to give the unsymmetrically-oriented calix[3]indole $\mathbf{2 5}$ in 30\% yield (Scheme 5). Furthermore, the 2-isomer $\mathbf{2 7}$ was reduced by sodium borohydride to the alcohol 30, which without purification on treatment with ethanol containing concentrated hydrochloric acid was converted into the symmetrically-oriented calix[3]indole $\mathbf{3 1}$ in 60\% yield (Scheme 5). Thus both calix[3]indole isomers were available for comparison and structural confirmation.

Similar treatment of the indole-7-carboxylic acid $\mathbf{1 5}$ with 4-tolualdehyde in ethanol containing concentrated hydrochloric acid gave the unsymmetrically-oriented calix[3]indole 32 in $40 \%$ yield. For the sake of comparison, the related symmetrically-oriented calix[3]indole $\mathbf{3 3}$ was obtained in $40 \%$ yield by the reaction of the indole 1 with 4-tolualdehyde in phosphoryl chloride: however in this case the unsymmetrically-oriented calix[3]indole $\mathbf{3 2}$ was also formed in $30 \%$ yield (Scheme 6 )<smiles>[R]c1c[nH]c2c(C(=O)O)c(OC)cc(OC)c12</smiles>

$15 \mathrm{R}=4-\mathrm{ClC}_{6} \mathrm{H}_{4}$

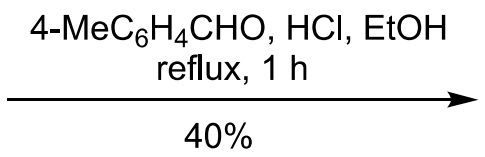

4- $\mathrm{MeC}_{6} \mathrm{H}_{4} \mathrm{CHO}, \mathrm{POCl}_{3}$ $\mathrm{CHCl}_{3}$, reflux, $1 \mathrm{~h}$

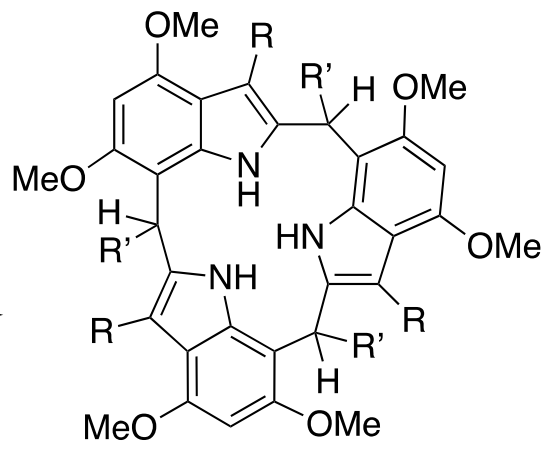

$+32(30 \%)$

$33 \mathrm{R}=4-\mathrm{ClC}_{6} \mathrm{H}_{4} \quad \mathrm{R}^{\prime}=4-\mathrm{MeC}_{6} \mathrm{H}_{4}$

$(40 \%)$

Scheme 6. Formation of calix[3]indoles 32 and 33.

Similar treatment of the indole-7-carboxylic acid $\mathbf{1 5}$ with piperonal in ethanol containing concentrated hydrochloric acid gave the unsymmetrically-oriented calix[3]indole $\mathbf{3 4}$ in 50\% yield (Scheme 7). In this case, the comparative reaction of the indole 1 with piperonal in phosphoryl chloride (conditions that usually favor formation of the symmetrically-oriented isomer) gave only the unsymmetrically-oriented calix[3]indole 34 in $84 \%$. This result shows the effect of a relatively slower reaction because of the less electrophilic aldehyde. 
<smiles>[R]c1c[nH]c2c(C(=O)O)c(OC)cc(OC)c12</smiles>

$15 \mathrm{R}=4-\mathrm{ClC}_{6} \mathrm{H}_{4}$<smiles>[R]c1c[nH]c2cc(OC)cc(OC)c12</smiles>

$1 \mathrm{R}=4-\mathrm{ClC}_{6} \mathrm{H}_{4}$

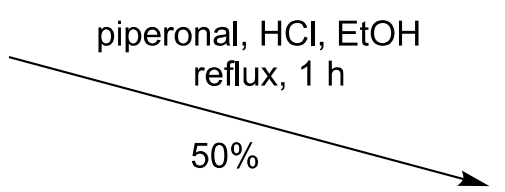

piperonal, $\mathrm{POCl}_{3}$ $\mathrm{CHCl}_{3}$, reflux, $1 \mathrm{~h}$

$84 \%$

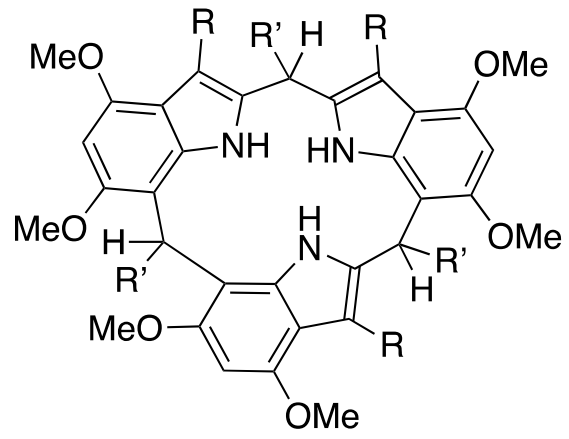

$34 \mathrm{R}=4-\mathrm{ClC}_{6} \mathrm{H}_{4} \quad \mathrm{R}^{\prime}=3,4-\mathrm{OCH}_{2} \mathrm{OC}_{6} \mathrm{H}_{3}$

Scheme 7. Formation of calix[3]indole 34.

\section{Reactions of indole carboxylic acids 16-18 with aryl aldehydes}

To explore the situation with an indole-2-carboxylic acid, two starting materials were available, carboxylic acids 16 and 17, with the latter being the more accessible. Both underwent reaction with 4-chlorobenzaldehyde in ethanol containing concentrated hydrochloric acid and gave the unsymmetrically-oriented calix[3]indoles 25 and $\mathbf{3 5}$ in 16 and 62\% yield, respectively (Scheme 8). These compounds were also formed respectively in 30 and $27 \%$ yield by reaction of the indoles 1 and $\mathbf{2}$ with 4-chlorobenzaldehyde catalyzed by chlorotrimethylsilane. Reaction of the indole-2,7-dicarboxylic acid $\mathbf{1 8}$ with 4-chlorobenzaldehyde in ethanol containing concentrated hydrochloric acid gave only a complex mixture. 


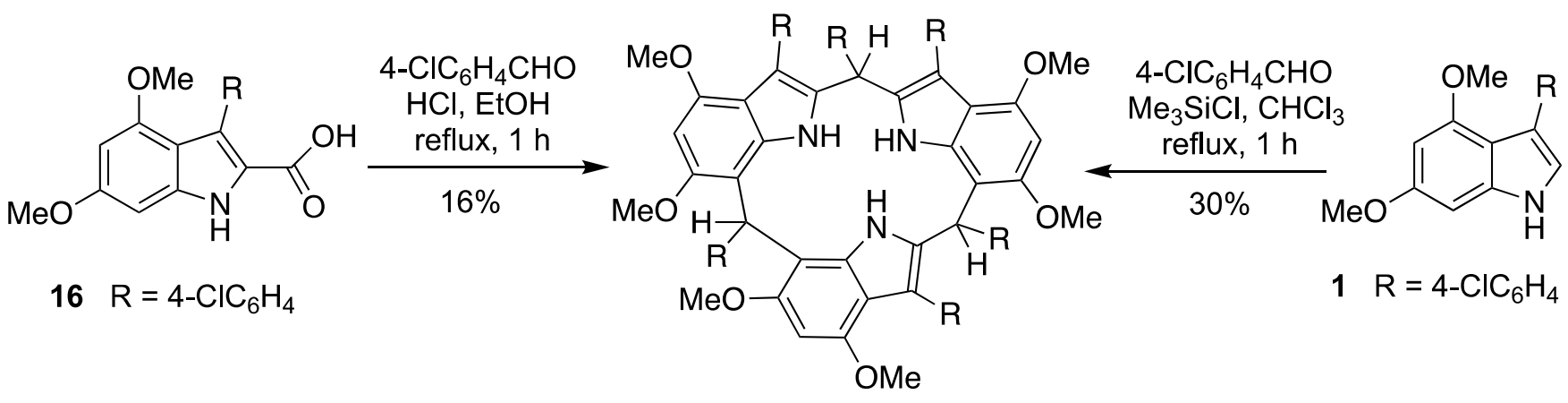

$25 \mathrm{R}=4-\mathrm{ClC}_{6} \mathrm{H}_{4}$

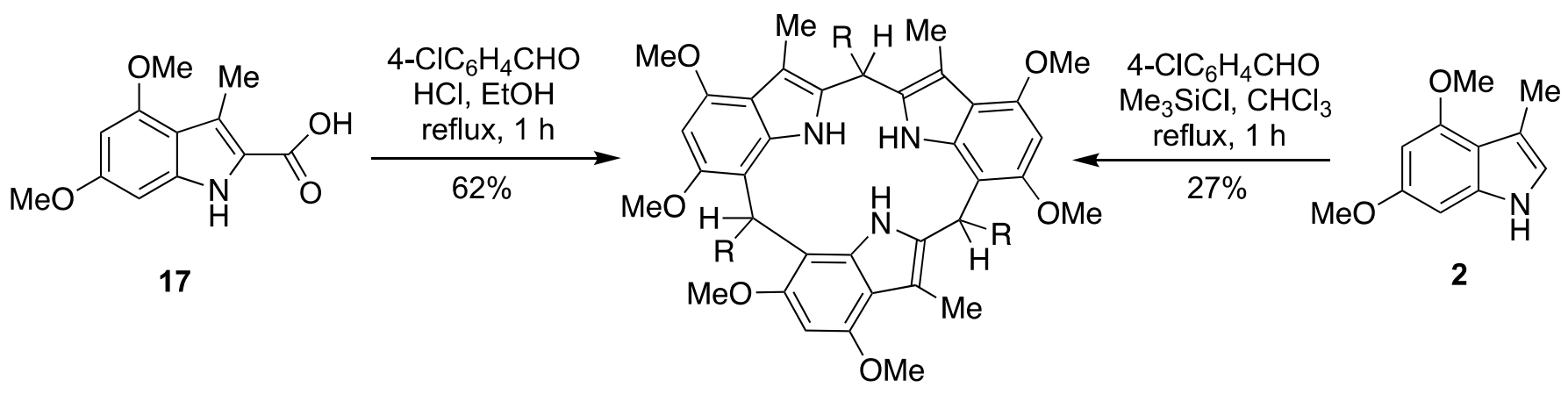

$35 \mathrm{R}=4-\mathrm{ClC}_{6} \mathrm{H}_{4}$<smiles>COc1cc(OC)c2c(C)c(C(=O)O)[nH]c2c1C(=O)O</smiles>

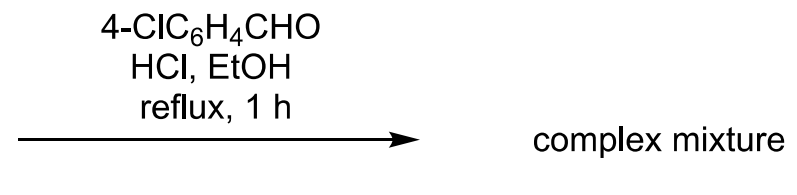

18

Scheme 8. Formation of calix[3]indoles 25 and $\mathbf{3 5 .}$

Reaction of hydroxymethylindole carboxylic acids 21 and 24 with acid

The crude hydroxymethylindole carboxylic acids $\mathbf{2 1}$ and $\mathbf{2 4}$ were submitted to a wide variety of acidic conditions but failed to generate clean reactions and isolable products. However, there was no evidence for the formation of any calix[3]indoles or calix[4]indoles, as were obtained from the related hydroxymethylindoles without carboxyl substituents. ${ }^{2}$

\section{Conclusions}

Acid-catalyzed reactions of 3-substituted 4,6-dimethoxyindole-7- and -2-carboxylic acids with aryl aldehydes generate unsymmetrically-oriented calix[3]indoles, with one 2,2'-linkage, one 2,7'-linkage and one 7,7'linkage, in a wide range of yields. This behavior contrasts with similar reactions of the parent indoles, which more commonly yield the symmetrically-oriented calix[3]indoles, with three 2,7'-linkages. The starting material carboxylic acids were prepared via the hydrolysis of trifluoroacetyl and trichloroacetyl substituents formed by acylation of the parent 3-substituted-4,6-dimethoxyindoles. These results expand the scope for the 
synthesis of calix[3]indoles by relatively simple one-pot acid-catalyzed reactions, and compliment other approaches involving linear sequences. The additional nucleophilic character of the 4,6-dimethoxyindoles leads to further new possibilities for synthesis of structurally diverse products.

\section{Experimental Section}

General. ${ }^{1} \mathrm{H}$ and ${ }^{13} \mathrm{C}$ NMR spectra were recorded on a Bruker $\mathrm{AC} 300 \mathrm{~F}\left({ }^{1} \mathrm{H}: 300 \mathrm{MHz},{ }^{13} \mathrm{C}: 75.5 \mathrm{MHz}\right)$ or a Bruker AM500 spectrometer. The chemical shifts $(\delta)$ and coupling constants $(J)$ are expressed in ppm and hertz, respectively. Carbon attribution $\mathrm{C}, \mathrm{CH}, \mathrm{CH}_{2}$ and $\mathrm{CH}_{3}$ were determined by ${ }^{13} \mathrm{C}, \mathrm{DEPT}$ and $\mathrm{HMQC}$ experiments. Infrared (IR) spectra were recorded on a Mattson Genesis Series FTIR spectrometer using potassium bromide disks, except where specified. Ultraviolet and visible (UV/Vis) spectra were recorded in tetrahydrofuran or methanol using a Carey 100 spectrometer. Mass spectra were recorded on a VG Quattro MS (EI) or a Finnigan MAT (MALDI). High resolution mass spectrometry (HRMS) was carried out at the Research School of Chemistry, Australian National University. Melting points were measured using a Mel-Temp melting point apparatus. Microanalyses were performed at the UNSW Microanalytical Unit and at the Campbell Microanalytical Laboratory, University of Otago, New Zealand. Column chromatography was carried out using Merck 230-400 mesh silica gel or Merck 70-230 mesh silica gel, whilst preparative TLC was performed using Merck 60GF 254 silica gel.

\section{Preparation of indole carboxylic acids}

3-(4-Chlorophenyl)-4,6-dimethoxy-7-trifluoroacetylindole (3) and 3-(4-chlorophenyl)-2,7-di(trifluoroacetyl)4,6-dimethoxyindole (7). A mixture of indole $1(0.30 \mathrm{~g}, 1.00 \mathrm{mmol})$ and trifluoroacetic anhydride (1.00 g, 7.10 $\mathrm{mmol})$ in dichloromethane $(20 \mathrm{~mL})$ was stirred at room temperature for $8 \mathrm{~h}$. Water was added and the organic layer was extracted with dichloromethane. The organic layer was washed with water until neutral, then dried $\left(\mathrm{MgSO}_{4}\right)$. The solvent was evaporated and the product purified by chromatography on silica gel using ethyl acetate/light petroleum (50:50) as eluent. The first fraction yielded compound $\mathbf{3}$ (140 $\mathrm{mg}, 35 \%$ ) as a yellow solid, $\mathrm{mp} 22{ }^{\circ} \mathrm{C}$ (from $\mathrm{CH}_{2} \mathrm{Cl}_{2} /$ light petroleum). IR ( $v_{\max } \mathrm{cm}^{-1}$ ): 3350, 1635, 1580, 1320, 1220, $1150,1090$. UV/Vis $\left(\lambda \max , \mathrm{nm}, \varepsilon, \mathrm{cm}^{-1} \mathrm{M}^{-1}\right): 203(44,000) .{ }^{1} \mathrm{H} \mathrm{NMR}\left(300 \mathrm{MHz}, \mathrm{CDCl}_{3}\right): \delta_{\mathrm{H}} 3.94,4.01$ (each 3H, 2s, OMe), 6.21 $(1 \mathrm{H}, \mathrm{s}, \mathrm{H} 5), 7.08(1 \mathrm{H}, \mathrm{d}, J 2.4 \mathrm{~Hz}, \mathrm{H} 2), 7.35-7.48(4 \mathrm{H}, \mathrm{m}, \mathrm{aryl}), 10.61(1 \mathrm{H}, \mathrm{br}, \mathrm{NH}) .{ }^{13} \mathrm{C} \mathrm{NMR}\left(75 \mathrm{MHz}, \mathrm{CDCl}_{3}\right): \delta_{\mathrm{c}}$ 55.6, 56.6 (OMe), 87.7 (C5), 100.1 ( $\left.\mathrm{CF}_{3}\right), 110.6,115.5,118.6,119.3$ (aryl C), 121.7 (C2), 127.9 (aryl CH), 130.7 (aryl C), 131.1 (aryl CH), 132.2, 133.6, 139.4 (aryl C), 162.5 (CO). MS (+El, m/z, \%): $385\left(\mathrm{M}+2, \mathrm{Cl}^{37 / 37}, 30\right), 383$ $\left(\mathrm{M}+1, \mathrm{Cl}^{35 / 35}, 90\right), 316$ (35), 314 (100). Anal. calcd for $\mathrm{C}_{18} \mathrm{H}_{13} \mathrm{ClF}_{3} \mathrm{NO}_{3}$ : C, 56.3; $\mathrm{H}, 3.4 ; \mathrm{N}, 3.7$. Found: $\mathrm{C}, 56.6 ; \mathrm{H}$, 3.7; N, 3.5\%. The second fraction yielded compound 7 (100 mg, 25\%) as a yellow solid, $\mathrm{mp} 200{ }^{\circ} \mathrm{C}(\mathrm{from}$ EtOAc). IR ( $\left.v_{\max } \mathrm{cm}^{-1}\right): 3400,1685,1640,1600,1590,1560,1330,1220,1190,1150,1030,990$. UV/Vis $\left(\lambda_{\max }\right.$, $\left.\mathrm{nm}, \varepsilon, \mathrm{cm}^{-1} \mathrm{M}^{-1}\right): 211$ (34,000), 252 (20,000), 346 (18,000). ${ }^{1} \mathrm{H}$ NMR $\left(300 \mathrm{MHz}^{\mathrm{CDCl}}{ }_{3}\right): \delta_{\mathrm{H}} 3.80,4.06$ (each $3 \mathrm{H}$, 2s, OMe), $6.16(1 \mathrm{H}, \mathrm{s}, \mathrm{H} 5), 7.26-7.35\left(4 \mathrm{H}, \mathrm{m}\right.$, aryl), $11.31(1 \mathrm{H}, \mathrm{br}, \mathrm{NH}) .{ }^{13} \mathrm{C} \mathrm{NMR}\left(75 \mathrm{MHz}, \mathrm{CDCl}_{3}\right): \delta_{\mathrm{C}} 56.0,56.8$ (OMe), 88.7 (C5), 99.4 ( $\left.\mathrm{CF}_{3}\right), 113.7,114.4,118.2,119.0,124.7$ (aryl C), 127.5 (aryl CH), 129.6, 131.1 (aryl C), 131.7 (aryl CH), 134.1, 140.3 (aryl C), 164.7, 166.4 (CO). MS (+El, m/z, \%): $481\left(\mathrm{M}+2, \mathrm{Cl}^{37 / 37}, 25\right), 479(\mathrm{M}+1$, $\left.\mathrm{Cl}^{35 / 35}, 80\right), 412$ (30), 410 (95), 69 (100). Anal. calcd for $\mathrm{C}_{20} \mathrm{H}_{12} \mathrm{ClF}_{6} \mathrm{NO}_{4}: \mathrm{C}, 50.1 ; \mathrm{H}, 2.5 ; \mathrm{N}, 2.9$. Found: $\mathrm{C}, 50.2 ; \mathrm{H}$, $2.4 ; \mathrm{N}, 2.8 \%$.

4,6-Dimethoxy-3-methyl-2-trifluoroacetylindole (6) and 4,6-dimethoxy-3-methyl-2,7-di(trifluoroacetyl)indole (8). A mixture of indole $2(0.50 \mathrm{~g}, 2.60 \mathrm{mmol})$ and trifluoroacetic anhydride $(2.60 \mathrm{~g}, 18.3 \mathrm{mmol})$ in dichloromethane $(20 \mathrm{~mL})$ was stirred at room temperature for $8 \mathrm{~h}$. Water was added and the organic layer was 
extracted with dichloromethane. The organic layer was washed with water until neutral, then dried $\left(\mathrm{MgSO}_{4}\right)$. The solvent was evaporated and the product purified by chromatography on silica gel using ethyl acetate/light petroleum (50:50) as eluent. The first fraction yielded compound 8 (50 mg, 5\%) as an orange solid, $\mathrm{mp} 180{ }^{\circ} \mathrm{C}$ (from $\mathrm{CH}_{2} \mathrm{Cl}_{2}$ /light petroleum). IR $\left(v_{\max }, \mathrm{cm}^{-1}\right)$ : 3420, 1650, 1590, 1510, 1340, 1240, 1200, 1160, 1100, 1020, 940. UV/Vis $\left(\lambda_{\max }, \mathrm{nm}, \varepsilon, \mathrm{cm}^{-1} \mathrm{M}^{-1}\right): 209$ (29,000), 251 (15,000), $348(12,000) .{ }^{1} \mathrm{H} \mathrm{NMR}\left(300 \mathrm{MHz}, \mathrm{CDCl}_{3}\right): \delta_{\mathrm{H}} 2.80$ $(3 \mathrm{H}, \mathrm{s}, \mathrm{Me}), 4.05,4.09$ (each 3H, 2s, OMe), $6.16(1 \mathrm{H}, \mathrm{s}, \mathrm{H} 5), 11.05(1 \mathrm{H}, \mathrm{br} \mathrm{s}, \mathrm{NH}) .{ }^{13} \mathrm{C} \mathrm{NMR}\left(75 \mathrm{MHz}, \mathrm{CDCl}_{3}\right): \delta_{\mathrm{c}}$ 12.5 (Me), 56.2, 56.7 (OMe), 88.1 (C5), $99.3\left(\mathrm{CF}_{3}\right), 114.8,115.2,118.6,119.0,124.8,130.7,140.7$ (aryl C), 165.7, 166.4 (CO). MS (+El, $m / z, \%): 384(\mathrm{M}+1,5), 383$ (M, 15), 315 (15), 314 (100). Anal. calcd for $\mathrm{C}_{15} \mathrm{H}_{11} \mathrm{~F}_{6} \mathrm{NO}_{4}$ : $\mathrm{C}, 47.0 ; \mathrm{H}, 2.9 ; \mathrm{N}, 3.7$. Found: $\mathrm{C}, 47.0 ; \mathrm{H}, 2.8 ; \mathrm{N}, 3.5 \%$. The second fraction yielded compound 6 (150 mg, $20 \%)$ as a yellow solid, $\mathrm{mp} 168{ }^{\circ} \mathrm{C}$ (from $\mathrm{CH}_{2} \mathrm{Cl}_{2} /$ light petroleum). IR ( $\left.\nu_{\max } \mathrm{cm}^{-1}\right)$ : 3360, 1710, 1600, 1570, 1410, 1320, 1260, 1250, 1150, 1070, 960. UV/Vis $\left(\lambda_{\max }, \mathrm{nm}, \varepsilon, \mathrm{cm}^{-1} \mathrm{M}^{-1}\right): 211(131,000), 253(43,000), 348(86,000) .{ }^{1} \mathrm{H} N M R$ (300 MHz, $\left.\mathrm{CDCl}_{3}\right): \delta_{\mathrm{H}} 2.80(3 \mathrm{H}, \mathrm{s}, \mathrm{Me}), 3.85,3.89$ (each 3H, 2s, OMe), $6.10(1 \mathrm{H}, \mathrm{d}, J 1.5 \mathrm{~Hz}, \mathrm{H} 5), 6.31$ (1H, d, J $1.5 \mathrm{~Hz}, \mathrm{H7}$ ), $8.58(1 \mathrm{H}, \mathrm{br} \mathrm{s}, \mathrm{NH}) .{ }^{13} \mathrm{C} \mathrm{NMR}\left(75 \mathrm{MHz}, \mathrm{CDCl}_{3}\right): \delta_{\mathrm{c}} 12.6$ (Me), 55.4, 55.6 (OMe), 85.3 (C5), 93.5 (C7), 114.7, 115.2, 119.0, 124.6, 140.7, 158.1 (aryl C), 162.9 (CO). MS (+El, m/z, \%): 288 (M+1, 15), 287 (M, 100$), 287$ (65). Anal. calcd for $\mathrm{C}_{13} \mathrm{H}_{12} \mathrm{~F}_{3} \mathrm{NO}_{3}$ : $\mathrm{C}, 54.4 ; \mathrm{H}, 4.2 ; \mathrm{N}, 4.9$. Found: $\mathrm{C}, 54.3 ; \mathrm{H}, 4.4 ; \mathrm{N}, 4.7 \%$.

\section{3-(4-Chlorophenyl)-4,6-dimethoxy-7-trichloroacetylindole (9) and 3-(4-chlorophenyl)-4,6-dimethoxy-2-} trichloroacetylindole (11). Trichloroacetylchloride $(2.00 \mathrm{~mL}, 17.9 \mathrm{mmol})$ was added dropwise to a solution of the indole $1(1.00 \mathrm{~g}, 3.50 \mathrm{mmol})$ in chloroform $(20 \mathrm{~mL})$. After completion of the addition, the solution was heated under reflux in $\mathrm{N}_{2}$ overnight. The mixture was allowed to cool to room temperature, and water (20 $\left.\mathrm{mL}\right)$ was added. The organic layer was extracted with dichloromethane $(2 \times 20 \mathrm{~mL})$ and the organic layer was dried $\left(\mathrm{MgSO}_{4}\right)$, and the solvent evaporated. Column chromatography of the residue using dichloromethane/light petroleum (50:50) as eluent gave, as the first fraction, compound 9 as an orange solid $(1.0 \mathrm{~g}, 66 \%), \mathrm{mp} 178{ }^{\circ} \mathrm{C}$ $\left(\mathrm{CH}_{2} \mathrm{Cl}_{2}\right.$ /light petroleum). IR $\left(\nu_{\max }, \mathrm{cm}^{-1}\right): 3380,1610,1580,1560,1340,1245,1215,1080$. UV/Vis $\left(\lambda_{\max }, \mathrm{nm}, \varepsilon\right.$, $\left.\mathrm{cm}^{-1} \mathrm{M}^{-1}\right): 212$ (14,000), 256 (12,600), 343 (8,000). ${ }^{1} \mathrm{H}$ NMR (300 MHz, CDCl $)$ ): $\delta_{\mathrm{H}} 3.43,3.99$ (each 3H, 2s, OMe), $6.23(1 \mathrm{H}, \mathrm{s}, \mathrm{H} 5), 7.08(1 \mathrm{H}, \mathrm{d}, J 2.0 \mathrm{~Hz}, \mathrm{H} 2), 7.35-7.48(4 \mathrm{H}, \mathrm{m}, \operatorname{aryl}), 10.29(1 \mathrm{H}, \mathrm{br} \mathrm{s}, \mathrm{NH}) .{ }^{13} \mathrm{C} \mathrm{NMR}(75 \mathrm{MHz}$, $\mathrm{CDCl}_{3}$ ): $\delta_{\mathrm{C}} 55.5,55.6$ (OMe), 87.7 (C5), $98.7\left(\mathrm{CCl}_{3}\right), 110.8,118.5,121.5$ (aryl C), 121.8 (C2), 127.8, 130.7, (aryl $\mathrm{CH}), 132.1,133.8,139.8,160.4,161.4$ (aryl C), 182.4 (CO). MS (+El, $m / z, \%): 435\left(\mathrm{M}+2, \mathrm{Cl}^{37 / 37}, 7\right), 433$ (M, $\left.\mathrm{Cl}^{35 / 35}, 15\right), 316$ (33), 314 (100). Anal. calcd for $\mathrm{C}_{18} \mathrm{H}_{13} \mathrm{Cl}_{4} \mathrm{NO}_{3}: \mathrm{C}, 49.9 ; \mathrm{H}, 3.0 ; \mathrm{N}$ 3.2. Found: $\mathrm{C}, 50.0 ; \mathrm{H}, 3.0 ; \mathrm{N}$, $3.1 \%$. The second fraction yielded compound 11 (300 mg, 17\%) as a yellow solid, mp $214{ }^{\circ} \mathrm{C}$ (from $\mathrm{CH}_{2} \mathrm{Cl}_{2} / \mathrm{light}$ petroleum). IR $\left(v_{\max }, \mathrm{cm}^{-1}\right)$ : 3400, 1670, 1615, 1570, 1380, 1350, 1250, 1210, 1150. UV/Vis $\left(\lambda_{\max }, \mathrm{nm}, \varepsilon, \mathrm{cm}^{-1} \mathrm{M}^{-}\right.$ $\left.{ }^{1}\right): 210$ (21,200), 281 (14,300), 360 (9,600). ${ }^{1} \mathrm{H}$ NMR (300 MHz, CDCl $): \delta_{\mathrm{H}} 3.63,3.88$ (each 3H, 2s, OMe), 6.13 $(1 \mathrm{H}, \mathrm{d}, J 2.0 \mathrm{~Hz}, \mathrm{H} 5), 6.45(1 \mathrm{H}, \mathrm{d}, J 2.0 \mathrm{~Hz}, \mathrm{H} 7), 7.36-7.48(4 \mathrm{H}, \mathrm{m}, \operatorname{aryl}), 8.95(1 \mathrm{H}, \mathrm{br}, \mathrm{NH}) .{ }^{13} \mathrm{C} \mathrm{NMR}(75 \mathrm{MHz}$, $\mathrm{CDCl}_{3}$ ): $\delta_{\mathrm{C}} 55.3,55.7$ (OMe), 87.6 (C5), 94.0 (C7), $96.3\left(\mathrm{CCl}_{3}\right), 113.2,120.7$ (aryl C), 127.3, 131.6 (aryl CH), 132.4, 132.9, 133.3, 139.1, 156.6, 161.5 (aryl C), 170.6 (CO). MS (+El, $m / z, \%): 435\left(\mathrm{M}+2, \mathrm{Cl}^{37 / 37}, 10\right), 433\left(\mathrm{M}^{+}, \mathrm{Cl}^{35 / 35}\right.$, 25), 314 (35), 279 (90), 264 (50), 150 (100). Anal. calcd for $\mathrm{C}_{18} \mathrm{H}_{13} \mathrm{Cl}_{4} \mathrm{NO}_{3}$ : C, 49.9; H, 3.0; N, 3.2. Found: C, 50.0; $\mathrm{H}, 2.9 ; \mathrm{N}, 3.3 \%$.

\section{4,6-Dimethoxy-3-methyl-2-trichloroacetylindole (12) and 4,6-dimethoxy-3-methyl-2,7-ditrichloroacetyl-} indole (14). The title compounds 12 and 14 were prepared according to the method of preparation of compound 9 using the indole $2(0.80 \mathrm{~g}, 4.19 \mathrm{mmol})$ and trichloroacetyl chloride $(2.30 \mathrm{~mL}, 21.0 \mathrm{mmol})$. The first fraction contained compound 14 as a green solid $(120 \mathrm{mg}, 6 \%), \mathrm{mp} 188{ }^{\circ} \mathrm{C}\left(\mathrm{MeOH} / \mathrm{CH}_{2} \mathrm{Cl}_{2}\right) . \mathrm{IR}\left(v_{\max }, \mathrm{cm}^{-1}\right)$ : $3380,1675,1615,1575,1500,1370,1355,1220,1170,1140,980$. UV/Vis $\left(\lambda_{\max }, \mathrm{nm}, \varepsilon, \mathrm{cm}^{-1} \mathrm{M}^{-1}\right): 208(5,800)$, 232 (4,900), 344 (4,800). ${ }^{1} \mathrm{H}$ NMR $\left(300 \mathrm{MHz}_{\mathrm{CDCl}}\right.$ ): $\delta_{\mathrm{H}} 2.48(3 \mathrm{H}, \mathrm{s}, \mathrm{Me}), 4.03,4.08$ (each 3H, 2s, OMe), 6.17 $(1 \mathrm{H}, \mathrm{s}, \mathrm{H} 5), 11.16(1 \mathrm{H}, \mathrm{br} \mathrm{s}, \mathrm{NH}) .{ }^{13} \mathrm{C} \mathrm{NMR}\left(75 \mathrm{MHz}, \mathrm{CDCl}_{3}\right): \delta_{\mathrm{C}} 13.2$ (Me), 55.8, 56.1 (OMe), 88.0 (C5), 96.0 (aryl C), 97.9, $98.2\left(\mathrm{CCl}_{3}\right), 113.9,122.2,132.5,140.3,164.2,164.3$ (aryl C), 172.0, 181.5 (CO). MS (+El, $\left.\mathrm{m} / \mathrm{z}, \%\right): 484$ 
$\left(\mathrm{M}+2, \mathrm{Cl}^{37 / 37}, 5\right), 483\left(\mathrm{M}+1, \mathrm{Cl}^{37 / 35}, 10\right), 481\left(\mathrm{M}, \mathrm{Cl}^{35 / 35}, 15\right), 377(80), 349$ (100). Anal. calcd for $\mathrm{C}_{15} \mathrm{H}_{11} \mathrm{Cl}_{6} \mathrm{NO}_{4}: \mathrm{C}_{\text {, }}$ 37.4; $\mathrm{H}, 2.3 ; \mathrm{N}, 2.9$. Found: $\mathrm{C}, 37.6 ; \mathrm{H}, 2.4 ; \mathrm{N}, 3.1 \%$. The second fraction contained compound 12 (300 mg, $21 \%)$ as a yellow solid, $\mathrm{mp} 178{ }^{\circ} \mathrm{C}\left(\mathrm{CH}_{2} \mathrm{Cl}_{2} /\right.$ light petroleum $)$. IR $\left(V_{\max } \mathrm{cm}^{-1}\right): 3395,1640,1620,1590,1500,1370$, 1320, 1260, 1220, 1200, 1150. UV/Vis $\left(\lambda_{\max }, \mathrm{nm}, \varepsilon, \mathrm{cm}^{-1} \mathrm{M}^{-1}\right): 213(22,600), 346(12,300), 253(8,000) .{ }^{1} \mathrm{H} N M R$ $\left(300 \mathrm{MHz}, \mathrm{CDCl}_{3}\right): \delta_{\mathrm{H}} 2.85(3 \mathrm{H}, \mathrm{s}, \mathrm{Me}), 3.85,3.90$ (each 3H, 2s, OMe), $6.12(1 \mathrm{H}, \mathrm{d}, J 1.5 \mathrm{~Hz}, \mathrm{H} 5), 6.35(1 \mathrm{H}, \mathrm{d}, J$ $1.5 \mathrm{~Hz}, \mathrm{H7}), 8.66\left(1 \mathrm{H}, \mathrm{br}\right.$ s, NH). ${ }^{13} \mathrm{C} \mathrm{NMR}\left(75 \mathrm{MHz}, \mathrm{CDCl}_{3}\right): \delta_{\mathrm{C}} 13.3$ (Me), 55.4, 55.8 (OMe), 85.5 (C5), 93.2 (C7), $96.5\left(\mathrm{CCl}_{3}\right), 114.0,121.3,133.3,139.6,157.6,162.4$ (aryl C), 171.2 (CO). $\mathrm{MS}(+\mathrm{El}, \mathrm{m} / \mathrm{z}, \%): 337\left(\mathrm{M}+2, \mathrm{Cl}{ }^{37 / 37}, 4\right)$, 335 (M, Cl35/35, 5), 274 (55), 272 (100). Anal. calcd for $\mathrm{C}_{13} \mathrm{H}_{12} \mathrm{Cl}_{3} \mathrm{NO}_{3}$ : C, 46.4; H, 3.6; N, 4.2. Found: C, 46.5; $\mathrm{H}$, $3.7 ; \mathrm{N}, 4.3 \%$.

3-(4-Chlorophenyl)-4,6-dimethoxyindole-7-carboxylic acid (15). Method a: A mixture of 7-trichloroacetylindole $9(50.0 \mathrm{mg}, 0.12 \mathrm{mmol})$ and $\mathrm{NaOH}(50.0 \mathrm{mg}, 1.25 \mathrm{mmol})$ was heated under reflux in ethanol/water (75:25; $25 \mathrm{~mL})$ for $1 \mathrm{~h}$. After cooling, the mixture was acidified with conc. $\mathrm{HCl}$ to afford compound 15 (32 $\mathrm{mg}$, $84 \%)$ as a colorless solid, $\mathrm{mp} 236{ }^{\circ} \mathrm{C}$ (EtOAc). IR $\left(\nu_{\max }, \mathrm{cm}^{-1}\right): 3360,1700,1580,1260,1210$. UV/Vis $\left(\lambda_{\max }, \mathrm{nm}, \varepsilon\right.$, $\left.\mathrm{cm}^{-1} \mathrm{M}^{-1}\right)$ : 239 (20,000), 214 (12,000), 310 (11,000). ${ }^{1} \mathrm{H}$ NMR (300 MHz, DMSO-d $\left.d_{6}\right): \delta_{\mathrm{H}} 3.87,3.94$ (each 3H, 2s, OMe), $6.46(1 \mathrm{H}, \mathrm{s}, \mathrm{H} 5), 7.19(1 \mathrm{H}, \mathrm{s}, \mathrm{H} 2)$ 7.37-7.51 (4H, m, aryl), $11.09(1 \mathrm{H}, \mathrm{br} \mathrm{s}, \mathrm{NH}), 12.06\left(1 \mathrm{H}, \mathrm{br} \mathrm{s}, \mathrm{CO}_{2} \mathrm{H}\right) .{ }^{13} \mathrm{C}$ NMR (75 MHz, $\mathrm{CDCl}_{3}$ ): $\delta_{\mathrm{C}}$ 55.6, 57.3 (OMe), 89.2 (C5), 110.0, 115.9 (aryl C), 123.6 (C2), 123.9 (aryl C), 127.7 (aryl CH), 130.3 (aryl C), 130.9 (aryl CH), 134.8, 138.4, 158.1, 159.1 (aryl C), 167.1 (CO). MS (+El, m/z, \%): 335 $\left(\mathrm{M}+2, \mathrm{Cl}^{37 / 37}, 5\right) 331\left(\mathrm{M}, \mathrm{Cl}^{35 / 35}, 20\right) 251$ (50), 177 (55). Anal. calcd for $\mathrm{C}_{17} \mathrm{H}_{14} \mathrm{ClNO}_{4}$ : C, 61.6; $\mathrm{H}, 4.3 ; \mathrm{N}, 4.2$. Found: C, $61.3 ; \mathrm{H}, 4.5 ; \mathrm{N}, 4.1 \%$.

Method b: A mixture of 7-trifluoroacetylindole $3(0.14 \mathrm{~g}, 0.36 \mathrm{mmol})$ and $\mathrm{KOH}(0.50 \mathrm{~g}, 8.90 \mathrm{mmol})$ was heated under reflux in ethanol/water $(75: 25,25 \mathrm{~mL})$ for $3 \mathrm{~h}$. After cooling, the mixture was acidified with conc. $\mathrm{HCl}$ to afford the title compound 15 (120 mg, 100\%) as a colorless solid.

3-(4-Chlorophenyl)-4,6-dimethoxyindole-2-carboxylic acid (16). Compound 16 was prepared according to Method a for preparation of compound 15 using indole $11(0.10 \mathrm{~g}, 0.23 \mathrm{mmol})$ and $\mathrm{NaOH}(1.00 \mathrm{~g}, 0.03 \mathrm{~mol})$ as a colorless solid (70 mg, 91\%), mp $232{ }^{\circ} \mathrm{C}(\mathrm{MeOH})$. IR $\left(v_{\max }, \mathrm{cm}^{-1}\right): 3400,1650,1630,1580,1520,1270,1200$, 1140. UV/Vis $\left(\lambda_{\max }, \mathrm{nm}, \varepsilon, \mathrm{cm}^{-1} \mathrm{M}^{-1}\right): 201(128,200), 249(91,800), 308(51,000) .{ }^{1} \mathrm{H}$ NMR $\left(300 \mathrm{MHz}, \mathrm{DMSO}-d_{6}\right)$ : $\delta_{\mathrm{H}}$ 3.56, 3.77 (each 3H, 2s, OMe), $6.12(1 \mathrm{H}, \mathrm{d}, J 2.0 \mathrm{~Hz}, \mathrm{H} 5), 6.50(1 \mathrm{H}, \mathrm{d}, J 2.0 \mathrm{~Hz}, \mathrm{H} 7), 7.33-7.34(4 \mathrm{H}, \mathrm{m}, \operatorname{aryl})$, $11.61\left(1 \mathrm{H}\right.$, br s, NH), $12.45\left(1 \mathrm{H}\right.$, br s, $\left.\mathrm{CO}_{2} \mathrm{H}\right) .{ }^{13} \mathrm{C}$ NMR $\left(75 \mathrm{MHz}, \mathrm{DMSO}-d_{6}\right): \delta_{\mathrm{C}} 55.1,55.4(\mathrm{OMe}), 86.7$ (C5), 92.7 (C7), 112.0, 121.4, 122.0 (aryl C), 126.5 (aryl CH), 131.1 (aryl C), 133.0 (aryl CH), 134.2, 138.0, 155.5, 159.2 (aryl C), 162.6 (CO). MS (+El, m/z, \%): $333\left(\mathrm{M}+2, \mathrm{Cl}^{37 / 37}, 30\right), 331\left(\mathrm{M}, \mathrm{Cl}^{35 / 35}, 95\right), 313$ (90), 287 (100). Anal. calcd for $\mathrm{C}_{17} \mathrm{H}_{14} \mathrm{ClNO}_{4}$ : $\mathrm{C}, 61.6 ; \mathrm{H}, 4.3 ; \mathrm{N}$ 4.2. Found: $\mathrm{C}, 61.2 ; \mathrm{H}, 4.3 ; \mathrm{N} 4.6 \%$.

4,6-Dimethoxy-3-methylindole-2-carboxylic acid (17). Compound 17 was prepared according to Method $b$ for preparation of compound 15 using indole $6(0.10 \mathrm{~g}, 0.34 \mathrm{mmol})$ and $\mathrm{KOH}(0.50 \mathrm{~g}, 0.87 \mathrm{mmol})$ as a colorless solid $(50 \mathrm{mg}, 61 \%), \mathrm{mp} 236^{\circ} \mathrm{C}$. IR $\left(v_{\max }, \mathrm{cm}^{-1}\right): 3380,1645,1590,1430,1290,1230,1205,1145,1110 .{ }^{1} \mathrm{H}$ NMR (300 MHz, DMSO-d6): $\delta_{\mathrm{H}} 2.62(3 \mathrm{H}, \mathrm{s}, \mathrm{Me}), 3.74,3.81$ (each 3H, 2s, OMe), $6.08(1 \mathrm{H}, \mathrm{d}, J 1.5 \mathrm{~Hz}, \mathrm{H} 5), 6.31(1 \mathrm{H}, \mathrm{d}$, J $1.5 \mathrm{~Hz}, \mathrm{H7}), 11.11\left(1 \mathrm{H}, \mathrm{br}\right.$ s, NH), $12.50\left(1 \mathrm{H}, \mathrm{br} \mathrm{s}, \mathrm{CO}_{2} \mathrm{H}\right) .{ }^{13} \mathrm{C}$ NMR (75 MHz, DMSO-d $)$ : $\delta_{\mathrm{c}} 12.1$ (Me), $55.3,55.4$ (OMe), 86.6 (C5), 91.9 (C7), 113.0, 119.3, 121.7, 156.3, 138.3, 159.2 (aryl C), 163.5 (CO). MS (+El, m/z, \%): 236 (M+1,15), 235 (100), 217 (75), 174 (45).

4,6-Dimethoxy-3-methylindole-2,7-dicarboxylic acid (18). Compound 18 was prepared according to the method of preparation of compound 17 using the indole $8(0.30 \mathrm{~g}, 0.78 \mathrm{mmol})$ and $\mathrm{KOH}(1.00 \mathrm{~g})$ as a colorless solid (180 mg, 82\%), $\mathrm{mp} 260^{\circ} \mathrm{C}$. IR $\left(v_{\max }, \mathrm{cm}^{-1}\right)$ : 3400, 3200, 1650, 1580, 1430, 1250, 1210, 1160, 970, 790, 760. ${ }^{1} \mathrm{H}$ NMR $\left(300 \mathrm{MHz}, \mathrm{CDCl}_{3}\right): \delta_{\mathrm{H}} 2.40(3 \mathrm{H}, \mathrm{s}, \mathrm{Me}), 3.98,4.11$ (each 3H, 2s, OMe), $6.20(1 \mathrm{H}, \mathrm{s}, \mathrm{H} 5), 6.84(1 \mathrm{H}, \mathrm{br} \mathrm{s}$, $\mathrm{NH}), 10.07,10.81$ (each $1 \mathrm{H}, 2 \mathrm{br} \mathrm{s}, \mathrm{CO}_{2} \mathrm{H}$ ). ${ }^{13} \mathrm{C} \mathrm{NMR}\left(75 \mathrm{MHz}, \mathrm{CDCl}_{3}\right): \delta_{\mathrm{c}} 11.9$ (Me), 55.5, 57.5 (OMe), 86.2 (C5), 105.0, 105.3, 112.1, 121.1, 138.7, 157.6, 159.9 (aryl C), 167.0 (CO). MS (+El, m/z, \%): 279 (M, 3 ), 235 (50), 217 
(100), 159 (50). Anal. calcd for $\mathrm{C}_{13} \mathrm{H}_{13} \mathrm{NO}_{6} \cdot 0.2 \mathrm{H}_{2} \mathrm{O}: \mathrm{C}, 55.2 ; \mathrm{H}, 4.8 ; \mathrm{N}, 4.9$. Found: $\mathrm{C}, 55.2 ; \mathrm{H}, 4.8 ; \mathrm{N}, 4.7 \%$.

\section{Preparation of indole hydroxy carboxylic acids}

3-(4-Chlorophenyl)-2-formyl-4,6-dimethoxy-7-trichloroacetylindole (19). A solution of the 7-trichloroacetylindole 9 ( $0.30 \mathrm{~g}, 0.70 \mathrm{mmol})$ and $\alpha, \alpha^{\prime}$-dichloromethyl methyl ether $(0.30 \mathrm{~mL}, 3.50 \mathrm{mmol})$ in chloroform (10 mL) was cooled at $0{ }^{\circ} \mathrm{C}$. Antimony pentachloride $(0.40 \mathrm{~mL}, 3.50 \mathrm{mmol})$ was added dropwise to the reaction mixture. After addition was complete, the reaction mixture was stirred at room temperature for $10 \mathrm{~min}, \mathrm{HCl}$ $(2 \mathrm{M}, 30 \mathrm{~mL})$ was then added, followed by water $(35 \mathrm{~mL})$. The organic layer was separated and dried $\left(\mathrm{MgSO}_{4}\right)$. The solvent was evaporated off and the residue chromatographed using dichloromethane as eluent to yield

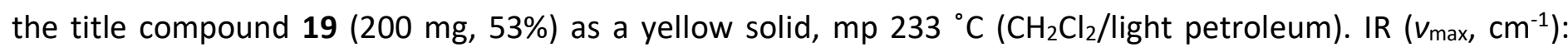
3380, 1640, 1560, 1520, 1200, 1150, 970, 810, 780, 710. UV/Vis $\left(\lambda_{\max }, \mathrm{nm}, \varepsilon, \mathrm{cm}^{-1} \mathrm{M}^{-1}\right): 204(38,200), 253$ $(19,800), 335$ (18,700). ${ }^{1} \mathrm{H}$ NMR $\left(300 \mathrm{MHz}, \mathrm{CDCl}_{3}\right): \delta_{\mathrm{H}} 3.87,4.02$ (each 3H, 2s, OMe), $6.21(1 \mathrm{H}, \mathrm{s}, \mathrm{H} 5), 7.42(4 \mathrm{H}$, s, aryl), $9.54(1 \mathrm{H}, \mathrm{s}, \mathrm{CHO}), 10.63(1 \mathrm{H}, \mathrm{br}, \mathrm{NH}) .{ }^{13} \mathrm{C} \mathrm{NMR}\left(75 \mathrm{MHz}, \mathrm{CDCl}_{3}\right): \delta_{\mathrm{c}} 55.8(\mathrm{OMe}), 88.4(\mathrm{C} 5), 97.9\left(\mathrm{CCl}_{3}\right)$, 98.6, 112.5 (aryl C), 127.9 (aryl CH), 128.7, 130.3, 131.8 (aryl C), 132.4 (aryl CH), 134.2, 140.3, 163.2, 163.3 (aryl C), 180.8, 182.2 (CO). MS (+El, m/z, \%): 461 (M, 10), 344 (30), 342 (100), 306 (25), 150 (80). Anal. calcd for $\mathrm{C}_{19} \mathrm{H}_{13} \mathrm{Cl}_{4} \mathrm{NO}_{4} \cdot 0.2 \mathrm{H}_{2} \mathrm{O}: \mathrm{C}, 49.1 ; \mathrm{H}, 2.9 ; \mathrm{N}, 3.0$. Found: $\mathrm{C}, 49.1 ; \mathrm{H}, 2.7 ; \mathrm{N}, 2.7 \%$.

3-(4-Chlorophenyl)-2-formyl-4,6-dimethoxyindole-7-carboxylic acid (20). According to the method of preparation of compound 16, reaction of indole $19(0.17 \mathrm{~g}, 0.37 \mathrm{mmol})$ with $\mathrm{NaOH}(1.0 \mathrm{~g})$ gave the title compound 20 (89 mg, 67\%) as a pale-yellow solid, $\mathrm{mp} 252{ }^{\circ} \mathrm{C}\left(\mathrm{CH}_{2} \mathrm{Cl}_{2} / \mathrm{MeOH}\right)$. IR $\left(v_{\max }, \mathrm{cm}^{-1}\right): 3400,3330,1700$, 1640, 1590, 1280, 1200, 980. UV/Vis $\left(\lambda_{\max }, \mathrm{nm}, \varepsilon, \mathrm{cm}^{-1} \mathrm{M}^{-1}\right): 256(21,700), 331(23,200), 288(9,400) .{ }^{1} \mathrm{H} N M R$ $\left(300 \mathrm{MHz}, \mathrm{CDCl}_{3}\right): \delta_{\mathrm{H}} 3.86,4.17$ (each $\left.3 \mathrm{H}, 2 \mathrm{~s}, \mathrm{OMe}\right), 6.25(1 \mathrm{H}, \mathrm{s}, \mathrm{H} 5), 7.41(4 \mathrm{H}, \mathrm{s}, \operatorname{aryl}), 9.57(1 \mathrm{H}, \mathrm{s}, \mathrm{CHO}), 10.50$ (1H, br s, NH), $10.99\left(1 \mathrm{H}\right.$, br s, $\left.\mathrm{CO}_{2} \mathrm{H}\right) .{ }^{13} \mathrm{C} \mathrm{NMR}\left(75 \mathrm{MHz} \mathrm{CDCl}_{3}\right): \delta_{\mathrm{c}} 55.7,57.6$ (OMe), 87.7 (C5), $94.5,112.8$ (aryl C), 127.8 (aryl CH), 128.0, 130.3, 132.4 (aryl C), 132.5 (aryl CH), 134.2, 139.5, 161.0, 161.5 (aryl C), 165.8, 181.1 (CO). MS (+El, m/z, \%): 361 (M, 10), 359 (20), 306 (35), 177 (60), 164 (90), 150 (100), 137 (85). Anal. calcd for $\mathrm{C}_{18} \mathrm{H}_{14} \mathrm{ClNO}_{5} \cdot 0.5 \mathrm{CH}_{2} \mathrm{Cl}_{2}$ : C, 55.2; $\mathrm{H}, 3.8 ; \mathrm{N}, 3.5$. Found: $\mathrm{C}, 55.6 ; \mathrm{H}, 3.7 ; \mathrm{N}, 3.2 \%$.

3-(4-Chlorophenyl)-2-hydroxymethyl-4,6-dimethoxyindole-7-carboxylic acid (21). Compound 20 (60.0 mg, $0.17 \mathrm{mmol}$ ) was partially dissolved in methanol $(25 \mathrm{~mL})$ and excess sodium borohydride $(62.0 \mathrm{mg}, 1.70 \mathrm{mmol})$ was added to the reaction mixture, which was then heated under reflux for $30 \mathrm{~min}$. Water (15 $\mathrm{mL})$ was added and the solution was then acidified with conc. $\mathrm{HCl}$ to yield a precipitate, which was filtered off and dried to yield the title compound 21 (40 mg, 65\%), mp $206^{\circ} \mathrm{C}$. IR $\left(v_{\max }, \mathrm{cm}^{-1}\right)$ : 3300, 1680, 1590, 1240, 1200, $1070,900$.

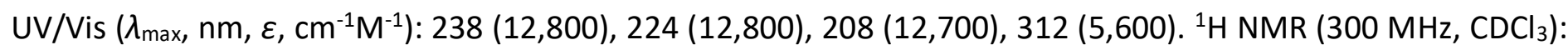
$\delta_{\mathrm{H}} 3.34(1 \mathrm{H}, \mathrm{s}, \mathrm{OH}), 3.83,4.14$ (each $\left.3 \mathrm{H}, 2 \mathrm{~s}, \mathrm{OMe}\right), 4.45\left(2 \mathrm{H}, \mathrm{s}, \mathrm{CH}_{2}\right), 6.24(1 \mathrm{H}, \mathrm{s}, \mathrm{H} 5), 7.33(4 \mathrm{H}, \mathrm{s}, \operatorname{aryl}), 10.58$ $\left(1 \mathrm{H}\right.$, br s, NH), $10.83\left(1 \mathrm{H}\right.$, br s, $\left.\mathrm{CO}_{2} \mathrm{H}\right) .{ }^{13} \mathrm{C} \mathrm{NMR}\left(75 \mathrm{MHz}, \mathrm{CDCl}_{3}\right): \delta_{\mathrm{c}} 55.4,57.5(\mathrm{OMe}), 58.2\left(\mathrm{CH}_{2}\right), 87.1(\mathrm{C} 5)$, 94.3, 112.4, 115.3 (aryl C), 127.5 (aryl CH), 131.8 (aryl C), 132.2 (aryl CH), 132.3, 133.1, 138.0, 157.7, 159.1 (aryl C), 166.7 (CO). Satisfactory microanalysis could not be obtained.

3-(4-Chlorophenyl)-7-formyl-4,6-dimethoxy-2-trichloroacetylindole (22). A solution of indole 12 (0.35 g, 0.81 $\mathrm{mmol})$ in trifluoroacetic acid $(2.5 \mathrm{~mL})$ was cooled in ice and triethyl orthoformate $(1.0 \mathrm{~mL})$ was added dropwise to the solution, which was stirred in an ice-cooled bath for $1 \mathrm{~h}$. Water $(30 \mathrm{~mL})$ was added and the mixture was extracted with dichloromethane, the extract dried $\left(\mathrm{MgSO}_{4}\right)$ and concentrated. The residue was chromatographed (dichloromethane as eluent) to give the title compound $\mathbf{2 2}$ (300 $\mathrm{mg}, \mathbf{8 0 \%}$ ) as a yellow solid, $\mathrm{mp} 234{ }^{\circ} \mathrm{C}\left(\mathrm{CH}_{2} \mathrm{Cl}_{2} /\right.$ light petroleum). IR $\left(v_{\max }, \mathrm{cm}^{-1}\right): 3400,1700,1650,1600,1520,1420,1360,1340,1250$, 1000. UV/Vis $\left(\lambda_{\max }, \mathrm{nm}, \varepsilon, \mathrm{cm}^{-1} \mathrm{M}^{-1}\right): 241(20,600), 215(14,000), 339(14,000), 288(8,000) .{ }^{1} \mathrm{H} \mathrm{NMR}(300 \mathrm{MHz}$, $\left.\mathrm{CDCl}_{3}\right): \delta_{\mathrm{H}} 3.78,4.02$ (each $\left.3 \mathrm{H}, 2 \mathrm{~s}, \mathrm{OMe}\right), 6.13(1 \mathrm{H}, \mathrm{s}, \mathrm{H} 5), 7.26-7.35(4 \mathrm{H}, \mathrm{m}, \operatorname{aryl}), 10.36(1 \mathrm{H}, \mathrm{s}, \mathrm{CHO}), 11.56(1$ $\mathrm{H}$, br s, NH). ${ }^{13} \mathrm{C} \mathrm{NMR}\left(75 \mathrm{MHz}, \mathrm{CDCl}_{3}\right): \delta_{\mathrm{C}} 55.8,56.5$ (OMe), 87.8 (C5), $95.9\left(\mathrm{CCl}_{3}\right), 103.7,112.4,122.0$ (aryl C), 127.5 (aryl CH), 131.2 (aryl C), 131.6 (aryl CH), 132.2, 133.6, 137.7, 163.7, 166.6 (aryl C), 171.3, 187.9 (CO). MS 
(+El, m/z, \%): 465 (M, Cl $\left.{ }^{37} / 37,3\right), 463$ (M, Cl37/35, 10), 461 (M, Cl $\left.{ }^{35} / 35,20\right), 459$ (15), 344 (30), 342 (100), 307 (60). Anal. calcd for $\mathrm{C}_{19} \mathrm{H}_{13} \mathrm{Cl}_{4} \mathrm{NO}_{4} \cdot 0.5 \mathrm{CH}_{2} \mathrm{Cl}_{2}$ : C, 46.5; $\mathrm{H}, 2.8 ; \mathrm{N}, 2.8$. Found: $\mathrm{C}, 46.1 ; \mathrm{H}, 2.7 ; \mathrm{N}, 2.9 \%$.

3-(4-Chlorophenyl)-7-formyl-4,6-dimethoxyindole-2-carboxylic acid (23). According to the method of preparation of compound 20, reaction of indole $22(0.20 \mathrm{~g}, 0.43 \mathrm{mmol})$ with $\mathrm{NaOH}(40.0 \mathrm{mg}, 1.00 \mathrm{mmol})$ gave compound 23 (130 mg, 83\%) as a colorless solid, $\mathrm{mp} 234{ }^{\circ} \mathrm{C}\left(\mathrm{CH}_{2} \mathrm{Cl}_{2} / \mathrm{MeOH}\right)$. IR $\left(v_{\max }, \mathrm{cm}^{-1}\right): 3450,1680,1650$, 1540, 1280, 1240, 1160, 990. UV/Vis $\left(\lambda_{\max }, \mathrm{nm}, \varepsilon, \mathrm{cm}^{-1} \mathrm{M}^{-1}\right): 247(31,000), 205(29,500), 336(18,000) .{ }^{1} \mathrm{H} N M R$ (300 MHz, DMSO- $\left.d_{6}\right): \delta_{\mathrm{H}} 3.79,4.01$ (each 3H, 2s, OMe), $6.44(1 \mathrm{H}, \mathrm{s}, \mathrm{H} 5), 7.36-7.37(4 \mathrm{H}, \mathrm{m}, \operatorname{aryl}), 10.25(1 \mathrm{H}, \mathrm{s}$, $\mathrm{CHO}), 10.70(1 \mathrm{H}, \mathrm{br} \mathrm{s}, \mathrm{NH}), 13.01\left(1 \mathrm{H}, \mathrm{br} \mathrm{s}, \mathrm{CO}_{2} \mathrm{H}\right) .{ }^{13} \mathrm{C}$ NMR $\left(75 \mathrm{MHz}, \mathrm{DMSO}-d_{6}\right): \delta_{\mathrm{C}} 56.3,57.4$ (OMe), 89.0 (C5), 126.8, 132.9 (aryl CH), 103.2, 111.6, 122.0, 123.1, 131.8, 132.8, 135.6, 161.9, 162.7 (aryl C), 165.1, 187.2 (CO). $\mathrm{MS}(+\mathrm{El}, \mathrm{m} / z, \%): 361$ (M, Cl$\left.{ }^{37 / 37}, 30\right), 359$ (M, Cl $\left.35 / 35,100\right), 314$ (7), 312 (30), 287 (20), 284 (30). Anal. calcd for $\mathrm{C}_{18} \mathrm{H}_{14} \mathrm{ClNO}_{5} \cdot 0.5 \mathrm{H}_{2} \mathrm{O}: \mathrm{C}, 58.6 ; \mathrm{H}, 4.1 ; \mathrm{N}$ 3.8. Found: $\mathrm{C}, 58.3 ; \mathrm{H}, 4.0 ; \mathrm{N}, 3.8 \%$.

3-(4-Chlorophenyl)-7-hydroxymethyl-4,6-dimethoxyindole-2-carboxylic acid (24). The indole 23 (0.10 g, 0.30 $\mathrm{mmol})$ in methanol $(15 \mathrm{~mL})$ was treated with sodium borohydride $(60.0 \mathrm{mg}, 1.60 \mathrm{mmol})$ and the mixture was heated under refluxed in $\mathrm{N}_{2}$ for $30 \mathrm{~min}$, allowed to cool to room temperature then diluted with water. The mixture was acidified with $\mathrm{HCl}(1 \mathrm{M})$ and the resulting precipitate was filtered and dried to give the title compound 24 (87 mg, 87\%), mp $296{ }^{\circ} \mathrm{C}\left(\mathrm{CH}_{2} \mathrm{Cl}_{2}-\mathrm{MeOH}\right)$. IR $\left(V_{\max }, \mathrm{cm}^{-1}\right): 3400,3320,1650,1620,1590,1260$, 1210, 1150, 1120, 990. UV/Vis $\left(\lambda_{\max }, \mathrm{nm}, \varepsilon, \mathrm{cm}^{-1} \mathrm{M}^{-1}\right): 206(8,500), 250(6,800), 313(3,000) .{ }^{1} \mathrm{H} \mathrm{NMR}(300 \mathrm{MHz}$, DMSO- $\left.d_{6}\right): \delta_{\mathrm{H}} 3.64,4.16$ (each 3H, 2s, OMe), $4.21\left(2 \mathrm{H}, \mathrm{s}, \mathrm{CH}_{2}\right), 6.51(1 \mathrm{H}, \mathrm{s}, \mathrm{H} 5), 7.33(4 \mathrm{H}, \mathrm{s}, \operatorname{aryl}), 10.47(1 \mathrm{H}, \mathrm{br}$ s, $\mathrm{NH}), 12.72\left(1 \mathrm{H}\right.$, br s, $\left.\mathrm{CO}_{2} \mathrm{H}\right)$. The $\mathrm{OH}$ signal was not observed. ${ }^{13} \mathrm{C} \mathrm{NMR}\left(75 \mathrm{MHz}, \mathrm{DMSO}-d_{6}\right): \delta_{\mathrm{C}} 17.9\left(\mathrm{CH}_{2}\right)$, 55.5, 57.3 (OMe), 89.4 (C5), 126.6, 132.9 (aryl CH), 102.4, 112.4, 121.9, 122.7, 131.3, 133.7, 136.6, 153.6, 154.5 (aryl C), 163.3 (CO). MS (+El, m/z, \%): 361 (M, Cl35/35, 5), 359 (15), 315 (70), 301 (80), 287 (100), 286 (55), 251 (100). Satisfactory microanalysis could not be obtained.

\section{Reactions of indole carboxylic acids and related compounds}

\section{2,4,10,12,18,24-Hexa(4-chlorophenyl)-6,8,14,20,22-hexamethoxy-25,28,30-triazaheptacyclo-}

[17.5.2.23,9.05,29.013,27.023,26]triaconta-1(24),3,5(29),6,8,11,13(27),14,16,19,21,23(26)-dodecaene (25). Method a: A mixture of indole $15(50.0 \mathrm{mg}, 0.15 \mathrm{mmol})$ and 4-chlorobenzaldehyde $(21.0 \mathrm{mg}, 0.15 \mathrm{mmol}) \mathrm{in}$ ethanol $(25 \mathrm{~mL})$ containing conc. $\mathrm{HCl}(1 \mathrm{~mL})$ was heated under reflux for $1 \mathrm{~h}$. The mixture was then allowed to cool to room temperature and the precipitate was filtered off and purified using chromatography with dichloromethane/light petroleum ether (50:50) as eluent to yield the title compound 25 (50 mg, 80\%), mp $>330{ }^{\circ} \mathrm{C}\left(\mathrm{CH}_{2} \mathrm{Cl}_{2} /\right.$ light petroleum); IR $\left(v_{\max }, \mathrm{cm}^{-1}\right)$ : 3420, 1620, 1600, 1350, 1270, 1220, 1100, 1000. ${ }^{1} \mathrm{H} \mathrm{NMR}(300$ $\left.\mathrm{MHz}, \mathrm{CDCl}_{3}\right): \delta_{\mathrm{H}} 3.46,3.65,3.69,3.78$ (each 3H, 4s, OMe), $3.71(6 \mathrm{H}, \mathrm{s}, \mathrm{OMe}), 5.34,5.74,6.47$ (each 1H, 3s, CH), 6.09, 6.17, 6.24 (each 1H, 3s, H5), $6.37(4 \mathrm{H}, \mathrm{s}, \operatorname{aryl}), 6.87-6.92(9 \mathrm{H}, \mathrm{m}, \operatorname{aryl}), 7.06-7.20(11 \mathrm{H}, \mathrm{m}, \operatorname{aryl}), 6.75,7.43$, 7.57 (each $1 \mathrm{H}, 3 \mathrm{~s}, \mathrm{NH}) .{ }^{13} \mathrm{C} \mathrm{NMR}\left(75 \mathrm{MHz}_{\mathrm{CDCl}}\right.$ ): $\delta_{\mathrm{c}} 37.6,39.2,39.7(\mathrm{CH}), 55.0,55.1,55.7,57.0,57.2,57.6$ (OMe), 89,4, 89.8, 90.1 (C5), 104.1, 105.8, 106.6, 113.5, 113.7, 114.6, 115.4 (aryl C), 127.1, 127.4, 127.5, 128.3, 128.7, 129.0, 131.6, 131.8, 131.9, 132.9, 133.3, 134.0 (aryl CH), 135.9, 136.3, 139.2, 139.4, 142.7, 153.3, 153.5, 154.1, 154.8, 155.0 (aryl C). MS (MALDI, $\mathrm{m} / z, \%$ ): 1231 (M, 60). Anal. calcd for $\mathrm{C}_{69} \mathrm{H}_{51} \mathrm{Cl}_{6} \mathrm{~N}_{3} \mathrm{O}_{6} \cdot 4.6 \mathrm{CH}_{2} \mathrm{Cl}_{2}$ : C, 54.5; $\mathrm{H}, 3.7 ; \mathrm{N}, 2.6$. Found: $\mathrm{C}, 54.5 ; \mathrm{H}, 3.7 ; \mathrm{N}, 3.0 \%$.

Method b: A mixture of indole $16(50.0 \mathrm{mg}, 0.15 \mathrm{mmol})$ and 4-chlorobenzaldehyde $(21.0 \mathrm{mg}, 0.15 \mathrm{mmol}) \mathrm{in}$ ethanol $(25 \mathrm{~mL})$ containing conc. $\mathrm{HCl}(1 \mathrm{~mL})$ was heated under reflux for $1 \mathrm{~h}$. The mixture was then allowed to cool to room temperature and the precipitate was filtered off and purified using chromatography with dichloromethane/light petroleum ether (50:50) as eluent to yield the title compound 25 (10 mg, 16\%).

Method c: To a mixture of indole $1(0.30 \mathrm{~g}, 1.00 \mathrm{mmol})$ and 4-chlorobenzaldehyde $(0.15 \mathrm{~g}, 1.30 \mathrm{mmol})$ in chloroform $(25 \mathrm{~mL})$ was added trichloromethylsilane $(0.20 \mathrm{~mL}, 1.70 \mathrm{mmol})$ and the mixture was heated under reflux for $1 \mathrm{~h}$. Cold water was added to the mixture and the chloroform extract washed with $4 \mathrm{M} \mathrm{NaOH}$ until 
the washings were basic, then dried $\left(\mathrm{MgSO}_{4}\right)$ and concentrated. The residue was chromatographed using dichloromethane/light petroleum (50:50) as eluent to yield compound 25 (130 $\mathrm{mg}, 30 \%$ ).

7-(4-Chlorobenzoyl)-3-(4-chlorophenyl)-4,6-dimethoxyindole (26) and 2-(4-chlorobenzoyl)-3-(4-chlorophenyl)-4,6-dimethoxyindole (27). Phosphoryl chloride $(0.50 \mathrm{~mL}, 5.60 \mathrm{mmol})$ was added to warm $\left(60{ }^{\circ} \mathrm{C}\right) 4-$ chloro- $N, N^{\prime}$-dimethylbenzamide $(2.00 \mathrm{~g}, 10.9 \mathrm{mmol})$ and the mixture was stirred for 5 min and indole 1 (1.50 $\mathrm{g}, 5.20 \mathrm{mmol}$ ) was added. The mixture was heated at $80{ }^{\circ} \mathrm{C}$ for $30 \mathrm{~min}$. After cooling, $\mathrm{NaOH}(2 \mathrm{M}, 100 \mathrm{~mL}) \mathrm{was}$ added and the mixture was extracted with dichloromethane $(3 \times 50 \mathrm{~mL})$. The organic layer was washed with $\mathrm{NaOH}(2 \mathrm{M}, 100 \mathrm{~mL})$, water, dried and concentrated. The residue was chromatographed using dichloromethane as eluent to afford as the first fraction, a yellow solid being the title compound 26 (1.40 g, $62 \%), \mathrm{mp} 208{ }^{\circ} \mathrm{C}\left(\mathrm{EtOH} / \mathrm{CH}_{2} \mathrm{Cl}_{2}\right)$. IR $\left(\nu_{\max }, \mathrm{cm}^{-1}\right): 3320,1620,1580,1350,1285,1130,1100$. UV/Vis $\left(\lambda_{\max }, \mathrm{nm}, \varepsilon\right.$, $\mathrm{cm}^{-1} \mathrm{M}^{-1}$ ): 266 (24,300), 373 (9,400). ${ }^{1} \mathrm{H}$ NMR (300 MHz, CDCl 3 ): $\delta_{\mathrm{H}} 3.64,3.92$ (each 3H, 2s, OMe), $6.21(1 \mathrm{H}, \mathrm{s}$, H5), $7.11(1 \mathrm{H}, \mathrm{d}, J 2.6 \mathrm{~Hz}, \mathrm{H} 2), 7.30-7.59\left(8 \mathrm{H}, \mathrm{m}\right.$, aryl), $10.29(1 \mathrm{H}, \mathrm{br} \mathrm{s}, \mathrm{NH}) .{ }^{13} \mathrm{C} \mathrm{NMR}\left(75 \mathrm{MHz}, \mathrm{CDCl}_{3}\right): \delta_{\mathrm{c}} 55.3$, 56.2 (OMe), 88.0 (C5), 103.8, 110.7, 117.8, 120.4 (aryl C), 121.8 (C2), 127.8, 127.9, 129.7, 130.7 (aryl CH), 131.8, 134.1, 136.7, 139.3, 140.7, 159.9 (aryl C), 194.6 (CO). MS (+El, $m / z, \%): 428\left(\mathrm{M}+2, \mathrm{Cl}^{37 / 37}, 15\right), 426(\mathrm{M}$, $\left.\mathrm{Cl}^{35 / 35}, 45\right), 111$ (100). Anal.calcd for $\mathrm{C}_{23} \mathrm{H}_{17} \mathrm{Cl}_{2} \mathrm{NO}_{3} \cdot 1.5 \mathrm{H}_{2} \mathrm{O}: \mathrm{C}, 60.9 ; \mathrm{H}, 4.4 ; \mathrm{N}, 3.1$. Found: $\mathrm{C}, 60.8 ; \mathrm{H}, 4.2 ; \mathrm{N}$, 2.9\%. The second fraction produced a yellow solid, being the title compound 27 (300 mg, 13\%), mp $236{ }^{\circ} \mathrm{C}$ $\left(\mathrm{EtOH} / \mathrm{CH}_{2} \mathrm{Cl}_{2}\right)$. IR $\left(v_{\max }, \mathrm{cm}^{-1}\right): 3360,1640,1605,1520,1420,1290,1230,1210,1140,1090$. UV/Vis $\left(\lambda_{\max }, \mathrm{nm}\right.$, $\left.\varepsilon, \mathrm{cm}^{-1} \mathrm{M}^{-1}\right): 265$ (23,400), $346(15,450) .{ }^{1} \mathrm{H}$ NMR $\left(300 \mathrm{MHz}, \mathrm{CDCl}_{3}\right): \delta_{\mathrm{H}} 3.67,3.88$ (each 3H, 2s, OMe), $6.16(1 \mathrm{H}$, d, J $2.0 \mathrm{~Hz}, \mathrm{H} 5), 6.47(1 \mathrm{H}, \mathrm{d}, J 2.0 \mathrm{~Hz}, \mathrm{H7}), 7.04-7.06(4 \mathrm{H}, \mathrm{m}, \operatorname{aryl}), 7.27-7.29(4 \mathrm{H}, \mathrm{m}, \operatorname{aryl}), 9.23(1 \mathrm{H}, \mathrm{br} \mathrm{s}, \mathrm{NH})$. ${ }^{13} \mathrm{C} \mathrm{NMR}\left(75 \mathrm{MHz}, \mathrm{CDCl}_{3}\right.$ ): $\delta_{\mathrm{C}} 55.1,55.7$ (OMe), 85.8 (C5), 93.5 (C7), 112.8, 125.1 (aryl C), 126.8, 127.8 (aryl CH), 130.0 (aryl C), $130.4(\operatorname{aryl} \mathrm{CH}), 132.8$ (aryl C), 133.0 (aryl CH), 136.3, 137.5, 138.9, 157.0, 161.4 (aryl C), 187.3 (CO). MS (+El, m/z, \%): $430\left(\mathrm{M}+4, \mathrm{Cl}^{37 / 37}, 10\right), 428\left(\mathrm{M}+2, \mathrm{Cl}^{37 / 37}, 55\right), 425$ (100). Anal. calcd for $\mathrm{C}_{23} \mathrm{H}_{17} \mathrm{Cl}_{2} \mathrm{NO}_{3} \cdot 0.5 \mathrm{CH}_{2} \mathrm{Cl}_{2}$ : C, 60.2; $\mathrm{H}, 3.8 ; \mathrm{N}, 3.0$. Found: $\mathrm{C}, 60.4 ; \mathrm{H}, 3.4 ; \mathrm{N}, 2.9 \%$.

4-Chlorophenyl-di-\{2-[7-(4-chlorobenzoyl)-3-(4-chlorophenyl)-4,6-dimethoxy]indolyl\}-methane (28). The mixture of indole $26(0.50 \mathrm{~g}, 1.20 \mathrm{mmol})$ and 4-chlorobenzaldehyde $(0.10 \mathrm{~g}, 0.60 \mathrm{mmol})$ in methanol $(25 \mathrm{~mL})$ containing conc. $\mathrm{HCl}(1.8 \mathrm{~mL})$ was heated under reflux for $3 \mathrm{~h}$. The resulting precipitate was filtered off, washed with water and dried to yield the title compound 28 (800 mg, 85\%) as a yellow solid, $\mathrm{mp} 268{ }^{\circ} \mathrm{C}$ $\left(\mathrm{MeOH} / \mathrm{CH}_{2} \mathrm{Cl}_{2}\right)$. IR $\left(v_{\max }, \mathrm{cm}^{-1}\right): 3400,1620,1590,1550,1490,1290,1220,1090,1020,990$. UV/Vis $\left(\lambda_{\max }, \mathrm{nm}\right.$, $\left.\varepsilon, \mathrm{cm}^{-1} \mathrm{M}^{-1}\right): 263$ (50,300), $333(21,400) .{ }^{1} \mathrm{H}$ NMR (300 MHz, CDCl $): \delta_{\mathrm{H}} 3.60,3.77$ (each 6H, 2s, OMe), $5.63(1 \mathrm{H}$, s, CH), $6.13(2 \mathrm{H}, \mathrm{s}, \mathrm{H} 5), 6.99-7.52\left(2 \mathrm{HH}, \mathrm{m}\right.$, aryl), $10.03(2 \mathrm{H}, \mathrm{br} \mathrm{s}, \mathrm{NH}) .{ }^{13} \mathrm{C} \mathrm{NMR}(75 \mathrm{MHz}, \mathrm{CDCl} 3): \delta_{\mathrm{C}} 25.4(\mathrm{CH})$, 55.3, 56.2 (OMe), 88.2 (C5), 114.6 (aryl C), 127.4, 127.7 (aryl CH), 129.4, 129.5 (aryl C), 129.8, 131.7 (aryl CH), 132.1, 132.7, 133.1, 138.2, 140.6, 159.5 (aryl C), 194.2 (CO). MS (+El, m/z, \%): 974 (M, Cl35/35, 7), 552 (30), 548 (100), 512 (40). Anal. calcd for $\mathrm{C}_{53} \mathrm{H}_{37} \mathrm{Cl}_{5} \mathrm{~N}_{2} \mathrm{O}_{6} \cdot 1.5 \mathrm{H}_{2} \mathrm{O}: \mathrm{C}, 63.5 ; \mathrm{H}, 4.0 ; \mathrm{N}, 2.8$. Found: $\mathrm{C}, 63.5 ; \mathrm{H}, 4.5 ; \mathrm{N}, 2.4 \%$.

4-Chlorophenyl-di-(2-\{3-(4-chlorophenyl)-7-[(4-chlorophenyl)hydroxymethyl]-4,6-dimethoxy\}indolyl)-

methane (29). Excess sodium borohydride $(0.50 \mathrm{~g}, 4.10 \mathrm{mmol})$ was added to a suspension of the indole 28 $(0.30 \mathrm{~g}, 0.30 \mathrm{mmol})$ in tetrahydrofuran $(25 \mathrm{~mL})$ and the mixture was heated under reflux for $30 \mathrm{~min}$. After cooling to room temperature, the solvent was evaporated to dryness, the residue was collected and washed with water to yield the title compound $29(420 \mathrm{mg}, 80 \%)$ as a colorless solid. ${ }^{1} \mathrm{H}$ NMR (300 MHz, DMSO- $\left.d_{6}\right): \delta_{\mathrm{H}}$ 3.61, 3.73 (each 6H, 2s, OMe), 5.49 (1H, s, CHPh), $6.13(2 \mathrm{H}, \mathrm{s}, \mathrm{CHO}), 6.26(2 \mathrm{H}, \mathrm{br} \mathrm{s}, \mathrm{OH}), 6.34(2 \mathrm{H}, \mathrm{s}, \mathrm{H} 5), 6.68-$ $6.97(8 \mathrm{H}, \mathrm{m}, \operatorname{aryl}), 7.07-7.20(4 \mathrm{H}, \mathrm{m}, \operatorname{aryl}), 7.23-7.39(8 \mathrm{H}, \mathrm{m}, \operatorname{aryl}), 10.13(2 \mathrm{H}, \mathrm{br} \mathrm{s}, \mathrm{NH}) .{ }^{13} \mathrm{C} \mathrm{NMR}(75 \mathrm{MHz}$, $\mathrm{CDCl}_{3}$ ): $\delta_{\mathrm{C}}$ 55.5, 57.2 (OMe), 60.6, 67.5 (CH), 90.3 (C5), 108.3, 111.6, 113.4 (aryl C), 127.4, 127.6 (aryl CH), 127.8 (aryl C), 128.0, 129.0 (aryl CH), 129.6 (aryl C), 130.9 (aryl CH), 131.0 (aryl C), 132.1 (aryl CH), 132.5, 134.1, $135.9,144.7,152.8,153.5,157.4$ (aryl C). Satisfactory microanalysis could not be obtained. 
1-(4-Chlorophenyl)-1-[3-(4-chlorophenyl)-4,6-dimethoxyindol-2-yl]methanol (30). Sodium borohydride (0.20 $\mathrm{g}, 4.10 \mathrm{mmol}$ ) was added to a suspension of the indole $27(0.20 \mathrm{~g}, 0.50 \mathrm{mmol})$ in absolute ethanol $(20 \mathrm{~mL})$. The mixture was heated under reflux for $30 \mathrm{~min}$ and after cooling to room temperature, the solvent was evaporated to dryness. The residue was collected and washed with water to yield the title compound 30 (180 $\mathrm{mg}, 89 \%)$, as a colorless solid, $\mathrm{mp} 170{ }^{\circ} \mathrm{C}$ (aqueous EtOH). IR $\left(v_{\max }, \mathrm{cm}^{-1}\right)$ : 3320-3300, 1600, 1200, 1150, 1010, 820, 780, 710. UV/Vis $\left(\lambda_{\max }, \mathrm{nm}, \varepsilon, \mathrm{cm}^{-1} \mathrm{M}^{-1}\right): 223$ (51,000), $276(18,000) .{ }^{1} \mathrm{H}$ NMR $\left(300 \mathrm{MHz}, \mathrm{DMSO}-d_{6}\right): \delta_{\mathrm{H}} 3.62$, 3.73 (each 3H, 2s, OMe), $5.68(1 \mathrm{H}, \mathrm{br} \mathrm{s}, \mathrm{OH}), 6.10(1 \mathrm{H}, \mathrm{d}, J 3.8 \mathrm{~Hz}, \mathrm{H} 5), 6.14(1 \mathrm{H}, \mathrm{s}, \mathrm{CH}), 6.48(1 \mathrm{H}, \mathrm{br} \mathrm{s}, \mathrm{H} 7), 7.23$ $\left(2 \mathrm{H}, \mathrm{d}, J 8.3 \mathrm{~Hz}\right.$, aryl), 7.33-7.39 (6H, m, aryl), $10.91(1 \mathrm{H}, \mathrm{br}, \mathrm{NH}) .{ }^{13} \mathrm{C}$ NMR $\left(75 \mathrm{MHz}, \mathrm{DMSO}-d_{6}\right): \delta_{\mathrm{C}} 55.4,55.7$ (OMe), 66.5 (CH), 87.8 (C5), 92.1 (C7), 103.1, 106.4, 112.5, 114.6 (aryl C), 127.6, 128.5, 130.8 (aryl CH), 131.8 (aryl C), 132.8 (aryl CH), 135.1, 135.6, 138.0, 156.5, 157.2 (aryl C). MS (+El, m/z, \%): $430(\mathrm{M}+2,7), 429(20), 427$ (30), 411 (40), 374 (100), 359 (20), 316 (20), 139 (55). Satisfactory microanalysis could not be obtained.

\section{2,4,10,12,18,20-Hexa(4-chlorophenyl)-6,8,14,16,22,24-hexamethoxy-26,28,30-triazaheptacyclo-}

[17.5.2.23,9.211,17.05,29.013,27.021,25)triaconta-1(24),3,5(29),6,8,11,13(27),14,16,19,21(25),22-dodecaene (31). The indole 30 (60.0 mg, $0.21 \mathrm{mmol})$ was partially dissolved in ethanol $(10 \mathrm{~mL})$ and conc. $\mathrm{HCl}$ (3 drops) was added to the mixture, which was stirred at room temperature for $1 \mathrm{~h}$, then diluted with water $(25 \mathrm{~mL})$. The product was extracted with dichloromethane $(3 \times 15 \mathrm{~mL})$, the organic layer was washed and dried $\left(\mathrm{MgSO}_{4}\right)$. The solvent was concentrated and the residue chromatographed using dichloromethane/light petroleum (50:50) as eluent to afford the title compound 31 (52 mg, 60\%), mp $270{ }^{\circ} \mathrm{C}$ (EtOAc/light petroleum). IR ( $v_{\text {max }}$ $\left.\mathrm{cm}^{-1}\right): 3540,1620,1600,1495,1350,1220,1160,1100,1020,1000$. UV/Vis $\left(\lambda_{\max }, \mathrm{nm}, \varepsilon, \mathrm{cm}^{-1} \mathrm{M}^{-1}\right): 302$ $(12,000) .{ }^{1} \mathrm{H}$ NMR $\left(300 \mathrm{MHz}, \mathrm{CDCl}_{3}\right): \delta_{\mathrm{H}} 3.55$ (6H, s, OMe), 3.72, 3.73, 3.88, 3.89 (each 3H, 4s, OMe), 5.84, 6.12, 6.46 (each $1 \mathrm{H}, 3 \mathrm{~s}, \mathrm{CH}), 6.23,6.36,6.39$ (each $1 \mathrm{H}, 3 \mathrm{~s}, \mathrm{H} 5), 6.49(2 \mathrm{H}, \mathrm{d}, J 8.2 \mathrm{~Hz}$, aryl), $6.57(2 \mathrm{H}, \mathrm{d}, J 8.2 \mathrm{~Hz}, \operatorname{aryl})$, $6.74(2 \mathrm{H}, \mathrm{d}, J 8.7 \mathrm{~Hz}$, aryl), 6.79-6.88 $(6 \mathrm{H}, \mathrm{m}, \operatorname{aryl}), 7.04-7.19(8 \mathrm{H}, \mathrm{m}, \operatorname{aryl}), 7.33(4 \mathrm{H}, \mathrm{s}, \operatorname{aryl}), 6.75,7.01,7.96$ (each $1 \mathrm{H}, 3 \mathrm{~s}, \mathrm{NH}) .{ }^{13} \mathrm{C} \mathrm{NMR}\left(75 \mathrm{MHz}, \mathrm{CDCl}_{3}\right): \delta_{\mathrm{C}} 36.1,37.8,38.9(\mathrm{CH}), 55.1,55.3,55.4,56.5,57.2,57.4(\mathrm{OMe})$, 89.2, 89.8 (C5), 104.4, 105.3, 106.7, 112.1, 113.6, 113.9, 114.7, 115.1 (aryl C), 127.1, 127.2 (aryl CH), 127.5, 128.2 (aryl C), 128.5, 128.7 (aryl CH), 129.1, 129.5, 129.9, 130.9 (aryl C), 131.4, 131.6 (aryl CH), 132.1, 132.9, 133.4, 134.1, 134.3, 134.5, 134.7, 134.8, 136.4, 137.6, 138.9, 139.9, 153.1, 153.6, 153.8, 154.0 (aryl C). MS (MALDI, $m / z, \%): 1230$ (M, 45). Anal. calcd for $\mathrm{C}_{69} \mathrm{H}_{51} \mathrm{Cl}_{6} \mathrm{~N}_{3} \mathrm{O}_{6}: \mathrm{C}, 67.3 ; \mathrm{H}, 4.2 ; \mathrm{N}, 3.4$. Found: $\mathrm{C}, 67.0 ; \mathrm{H}, 4.0 ; \mathrm{N}$, $3.1 \%$.

\section{4,12,24-Tri(4-chlorophenyl)-6,8,14,20,22-hexamethoxy-2,10,18-tri-(4-tolyl)-25,28,30-triazaheptacyclo-} [17.5.2.23,9.05,29.013,27.023,26] triaconta-1(24),3,5(29),6,8,11,13(27),14,16,19,21,23(26)-dodecaene (32). The mixture of indole $15(75.0 \mathrm{mg}, 0.22 \mathrm{mmol})$ and 4-tolualdehyde $(27.0 \mathrm{mg}, 0.22 \mathrm{mmol})$ in ethanol $(25 \mathrm{~mL})$ containing concentrated $\mathrm{HCl}(1 \mathrm{~mL})$ was heated under reflux for $1 \mathrm{~h}$. After cooling, the precipitate was filtered off and chromatographed using dichloromethane/light petroleum (50:50) as eluent to yield the title compound 32 (40 mg, 40\%), $\mathrm{mp}>330{ }^{\circ} \mathrm{C}$ (EtOAc/light petroleum). IR $\left(V_{\max }, \mathrm{cm}^{-1}\right): 3410,1620,1595,1575$, 1490, 1420, 1340, 1210, 1150, 1100, 1000. UV/Vis $\left(\lambda_{\max }, \mathrm{nm}, \varepsilon, \mathrm{cm}^{-1} \mathrm{M}^{-1}\right): 297(7,000) .{ }^{1} \mathrm{H} \mathrm{NMR}(300 \mathrm{MHz}$, $\mathrm{CDCl}_{3}$ ): $\delta_{\mathrm{H}} 2.18,2.21,2.35$ (each 3H, 3s, Me), 3.37, 3.60, 3.64, 3.65, 3.67, 3.77 (each 3H, 6s, OMe), 5.37, 5.72, 6.49, (each 1H, 3s, CH), 6.09, 6.11, 6.77 (each $1 \mathrm{H}, 3 \mathrm{~s}, \mathrm{H5}), 6.32(4 \mathrm{H}, \mathrm{d}, J 8.2 \mathrm{~Hz}$, aryl), $6.68(2 \mathrm{H}, \mathrm{d}, J 7.2 \mathrm{~Hz}, \operatorname{aryl})$, $6.78(2 \mathrm{H}, \mathrm{d}$, J $8.2 \mathrm{~Hz}, \operatorname{aryl}), 6.82-6.97(7 \mathrm{H}, \mathrm{m}, \operatorname{aryl}), 7.04-7.15(9 \mathrm{H}, \mathrm{m}$, aryl), 7.34, 7.40, 7.42 (each 1H, $3 \mathrm{br}, \mathrm{NH}$ ). ${ }^{13} \mathrm{C}$ NMR $\left(75 \mathrm{MHz}_{\mathrm{CDCl}}\right): \delta_{\mathrm{C}} 21.1,21.2,21.4(\mathrm{Me}), 36.1,37.8,38.9(\mathrm{CH}), 54.7,55.1,55.2,57.4,57.6,57.9$ (OMe), 90.1, 90.5, 90.8 (C5), 106.0, 107.8, 112.1, 112.5, 113.0, 114.0, 114.1, 114.8 (aryl C), 124.8, 127.0, 127.1, 127.3, 128.0, 128.9, 129.0, 129.2, 129.5 (aryl CH), 132.6, 133.5, 133.8, 135.4, 135.6, 136.0, 136.3, 137.7, 138.0, 138.1, 141.0, 152.9, 153.0, 153.3, 153.7, 154.6, 154.8 (aryl C). MS (MALDI, m/z, \%): 1169 (M, 60). Anal. calcd for $\mathrm{C}_{72} \mathrm{H}_{60} \mathrm{Cl}_{3} \mathrm{~N}_{3} \mathrm{O}_{6} \cdot 1.0 \mathrm{H}_{2} \mathrm{O}: \mathrm{C}, 72.8 ; \mathrm{H}, 5.3 ; \mathrm{N}, 3.5$. Found: $\mathrm{C}, 72.6 ; \mathrm{H}, 5.5 ; \mathrm{N}, 3.4 \%$. 
4,12,24-Tri-(4-chlorophenyl)-6,8,14,20,22-hexamethoxy-2,10,18-tri-(4-tolyl)-25,28,30-triazaheptacyclo$[17.5 .2 .23,9.05,29.013,27.023,26]$ triaconta-1(24),3,5(29),6,8,11,13(27),14,16,19,21,23(26)dodecaene and 2,10,18-Tri-(4-chlorophenyl)-4,12,20-tri(4-tolyl)-6,8,14,16,22,24-hexamethoxy-26,28,30-triazaheptacyclo[17.5.2.23,9.211,17.05,29.013,27.021,25)triaconta-1(24),3,5(29),6,8, $\quad 11,13(27), 14,16,19,21(25), 22-$ dodecaene (33). Phosphoryl chloride $(0.20 \mathrm{~mL}, 2.20 \mathrm{mmol})$ was added to an ice-cold solution of indole 1 (0.30 $\mathrm{g}, 1.10 \mathrm{mmol})$ and 4-tolualdehyde $(0.13 \mathrm{~g}, 1.10 \mathrm{mmol})$ in dry chloroform $(15 \mathrm{~mL})$ and the mixture was heated under reflux for $1 \mathrm{~h}$. Cold water $(15 \mathrm{~mL})$ was added and the chloroform extract was dried $\left(\mathrm{MgSO}_{4}\right)$ and concentrated to give a residue which was chromatographed using dichloromethane/light petroleum (50:50) as eluent. The first fraction gave the symmetrically linked calix[3]indole 33 (160 mg, 40\%), mp $140{ }^{\circ} \mathrm{C}$ (EtOAc). IR $\left(v_{\max }, \mathrm{cm}^{-1}\right): 3410,1730,1620,1600,1515,1490,1340,1275,1210,1150,1090,1000$. UV/Vis $\left(\lambda_{\max }, \mathrm{nm}, \varepsilon, \mathrm{cm}^{-}\right.$ ${ }^{1} \mathrm{M}^{-1}$ ): 289 (58,100). ${ }^{1} \mathrm{H}$ NMR (300 MHz, $\left.\mathrm{CDCl}_{3}\right): \delta_{\mathrm{H}}$ 2.08, 2.27, 2.38 (each 3H, 3s, Me), 3.52, 3.54, 3.69, 3.70, 3.77, 3.78 (each 3H, 6s, OMe), $5.89(1 \mathrm{H}, \mathrm{s}, \mathrm{CH}), 6.11(2 \mathrm{H}, \mathrm{s}, \mathrm{H} 5+\mathrm{CH}), 6.22(1 \mathrm{H}, \mathrm{s}, \mathrm{H} 5), 6.23(\mathrm{~s} 1 \mathrm{H}, \mathrm{CH}), 6.26(1 \mathrm{H}, \mathrm{s}$, H5), 6.37-6.39 (4H, m, aryl), $6.59(2 \mathrm{H}, \mathrm{d}, J 7.7 \mathrm{~Hz}, \operatorname{aryl}), 6.73(2 \mathrm{H}, \mathrm{d}, J 8.2 \mathrm{~Hz}, \operatorname{aryl}), 6.80(2 \mathrm{H}, \mathrm{d}, J 8.2 \mathrm{~Hz}, \operatorname{aryl})$, 6.85-6.97 (6H, m, aryl), $7.00(2 \mathrm{H}, \mathrm{s}, \mathrm{NH}), 7.01-7.13(4 \mathrm{H}, \mathrm{m}, \operatorname{aryl}), 7.30-7.36(4 \mathrm{H}, \mathrm{m}, \operatorname{aryl}), 8.08(1 \mathrm{H}, \mathrm{s}, \mathrm{NH}) .{ }^{13} \mathrm{C}$ NMR (75 MHz, CDCl $)$ : $\delta_{c}$ 20.9, $21.3(\mathrm{Me}), 36.6,38.8,39.0(\mathrm{CH}), 55.2,55.4,56.9,57.3,57.9$ (OMe), 89.4, 89.8, 90.5 (C5), 106.0, 106.7, 107.7, 112.1, 113.5, 113.8, 114.2, 114.6 (aryl C), 126.4, 126.9, 127.0, 127.1, 127.5, 128.4, 128.5, 129.3, 129.7 (aryl CH), 130.9, 131.4 (aryl C), 131.8, 132.0, 132.2 (aryl CH), 134.0, 134.3, 134.6, 134.9, 135.1, 135.4, 136.1, 136.3, 136.5, 137.3, 138.5, 152.8, 153.2, 153.7 (aryl C). MS (MALDI, m/z, \%): 1169 (M, 60). Anal. calcd for $\mathrm{C}_{72} \mathrm{H}_{60} \mathrm{Cl}_{3} \mathrm{~N}_{3} \mathrm{O}_{6} \cdot 0.5 \mathrm{H}_{2} \mathrm{O}: \mathrm{C}, 73.4 ; \mathrm{H}, 5.2 ; \mathrm{N}, 3.6$. Found: $\mathrm{C}, 73.5 ; \mathrm{H}, 5.5 ; \mathrm{N}, 3.3 \%$. The second fraction gave compound 32 (120 mg, 30\%), $\mathrm{mp}>330{ }^{\circ} \mathrm{C}$ (EtOAc/light petroleum).

4,12,24-Tri-(4-chlorophenyl)-6,8,14,20,22-hexamethoxy-2,10,18-tripiperonyl-25,28,30-triazaheptacyclo[17.5.2.23,9.05,29.013,27.023,26]triaconta-1(24),3,5(29),6,8,11,13(27),14,16,19,21,23(26)-dodecaene

Method a: The mixture of indole $15(60.0 \mathrm{mg}, 0.18 \mathrm{mmol})$ and piperonal $(27.0 \mathrm{mg}, 0.22 \mathrm{mmol})$ in ethanol (25 $\mathrm{mL}$ ) containing concentrated $\mathrm{HCl}(1 \mathrm{~mL})$ was heated under reflux for $1 \mathrm{~h}$. After cooling, the precipitate was filtered off and chromatographed using dichloromethane as eluent to yield the title compound 34 (38.0 mg, $50 \%)$, as a pale colorless solid, $\mathrm{mp} 256{ }^{\circ} \mathrm{C}$ (EtOAc/light petroleum). IR $\left(v_{\max }, \mathrm{cm}^{-1}\right): 3460,3420,1745,1635$, 1600, 1490, 1440, 1345, 1240, 1220, 1050, 1000. UV/Vis $\left(\lambda_{\max }, \mathrm{nm}, \varepsilon, \mathrm{cm}^{-1} \mathrm{M}^{-1}\right): 204(30,700), 292(11,700) .{ }^{1} \mathrm{H}$ NMR $\left(300 \mathrm{MHz}_{\mathrm{CDCl}}\right.$ ): $\delta_{\mathrm{H}} 3.43,3.61,3.73,3.75$ (each $\left.3 \mathrm{H}, 4 \mathrm{~s}, \mathrm{OMe}\right), 3.69(6 \mathrm{H}, \mathrm{s}, \mathrm{OMe}), 5.30,5.64,6.42$ (each $1 \mathrm{H}, 3 \mathrm{~s}, \mathrm{CH}), 5.82\left(4 \mathrm{H}, \mathrm{s}, \mathrm{CH}_{2}\right), 5.88\left(2 \mathrm{H}, \mathrm{s}, \mathrm{CH}_{2}\right), 6.11,6.17,6.37$ (each $\left.1 \mathrm{H}, 3 \mathrm{~s}, \mathrm{H} 5\right), 6.01-6.12(4 \mathrm{H}, \mathrm{m}$, aryl), 6.26$6.31(4 \mathrm{H}, \mathrm{m}, \operatorname{aryl}), 6.43-6.57(4 \mathrm{H}, \mathrm{m}, \operatorname{aryl}), 7.04-7.16(9 \mathrm{H}, \mathrm{m}, \operatorname{aryl}), 6.29,6.97,7.38(3 \mathrm{H}, \mathrm{s}, \mathrm{NH}) .{ }^{13} \mathrm{C} \mathrm{NMR}(75$ $\left.\mathrm{MHz}, \mathrm{CDCl}_{3}\right): \delta_{\mathrm{C}} 37.5,38.7,40.2(\mathrm{CH}), 54.6,55.1,55.2,57.4,57.8$ (OMe), 89.8, 90.4, 90.8 (C5), 100.8, 100.9, 101.2 ( $\left.\mathrm{CH}_{2} \mathrm{O}\right), 106.3,107.5,107.7,108.2,109.4,111.7,114.9,120.1$ (aryl C), 127.1, 127.3, 127.4, $131.8,131.9$ (aryl CH), 133.2, 133.5, 134.3, 135.6, 136.4, 137.6, 146.0, 147.6, 147.9, 153.5, 154.6, 155.1 (aryl C). MS (MALDI, m/z, \%): 1258 (M, 60). Anal. calcd for $\mathrm{C}_{72} \mathrm{H}_{54} \mathrm{Cl}_{3} \mathrm{~N}_{3} \mathrm{O}_{12} \cdot 4.0 \mathrm{H}_{2} \mathrm{O}: \mathrm{C}, 64.9 ; \mathrm{H}, 4.7 ; \mathrm{N}, 3.2$. Found: $\mathrm{C}, 64.9 ; \mathrm{H}, 4.2 ; \mathrm{N}$, $3.1 \%$.

Method b: Phosphoryl chloride $(0.20 \mathrm{~mL}, 2.20 \mathrm{mmol})$ was added to an ice-cold solution of indole 1 (0.30 g, $1.10 \mathrm{mmol})$ and piperonal $(0.16 \mathrm{~g}, 1.00 \mathrm{mmol})$ in dry chloroform $(15 \mathrm{~mL})$ and the mixture was heated under reflux for $1 \mathrm{~h}$. Cold water $(15 \mathrm{~mL})$ was added and the chloroform extract was dried $\left(\mathrm{MgSO}_{4}\right)$ and concentrated to give a residue which was chromatographed using dichloromethane as eluent to produce compound 34 (360 $\mathrm{mg}, 84 \%)$.

2,10,18-Tri-(4-chlorophenyl)-6,8,14,20,22-hexamethoxy-4,12,24-trimethyl-25,28,30-triazaheptacyclo[17.5.2.23,9.05,29.013,27.023,26]triaconta-1(24),3,5(29),6,8,11,13(27),14,16,19,21,23(26)-dodecaene

Method a: The mixture of indole $17(30.0 \mathrm{mg}, 0.13 \mathrm{mmol})$ and 4-chlorobenzaldehyde $(17.8 \mathrm{mg}, 0.13 \mathrm{mmol}) \mathrm{in}$ ethanol $(25 \mathrm{~mL})$ containing concentrated $\mathrm{HCl}(1 \mathrm{~mL})$ was heated under reflux for $1 \mathrm{~h}$. After cooling, the 
precipitate was filtered off and chromatographed using dichloromethane-light petroleum (50:50) as eluent to yield the title compound 34 (25 mg, 62\%), as a pale colorless solid, $\mathrm{mp} 214{ }^{\circ} \mathrm{C}\left(\mathrm{CH}_{2} \mathrm{Cl}_{2} /\right.$ light petroleum). IR $\left(v_{\max }, \mathrm{cm}^{-1}\right): 3420,3400,1590,1570,1330,1320,1220,1200,1155,1120,1080,980,800$. UV/Vis $\left(\lambda_{\max }, \mathrm{nm}, \varepsilon\right.$, $\mathrm{cm}^{-1} \mathrm{M}^{-1}$ ): 232 (22,200), 285 (15,400). ${ }^{1} \mathrm{H}$ NMR (300 MHz, CDCl $)$ : $\delta_{\mathrm{H}}$ 1.99, 2.34, 2.48 (each 3H, 3s, Me), 3.57, 3.60, 3.65, 3.85, 3.88, 3.93 (each 3H, 6s, OMe), 5.44, 5.87 (each 1H, 2s, CH), 5.98, 6.21, 6.31 (each 1H, 3s, H5), $6.25(1 \mathrm{H}, \mathrm{d}, J 8.6 \mathrm{~Hz}, \operatorname{aryl}), 6.30(1 \mathrm{H}, \mathrm{s}, \operatorname{aryl}), 6.34(2 \mathrm{H}, \mathrm{s}, \mathrm{CH}+\operatorname{aryl}), 6.62-6.65(2 \mathrm{H}, \mathrm{m}, \operatorname{aryl}), 6.71(2 \mathrm{H}, \mathrm{d}, J 7.5 \mathrm{~Hz}$, aryl), 6.95-7.01 (3H, m, aryl), $7.13\left(2 \mathrm{H}, \mathrm{d}, J 7.9 \mathrm{~Hz}\right.$, aryl), 6.41, 7.08, 7.52 (each $1 \mathrm{H}, 3 \mathrm{br}$ s, NH). ${ }^{13} \mathrm{C} \mathrm{NMR}(75$ $\left.\mathrm{MHz}, \mathrm{CDCl}_{3}\right): \delta_{\mathrm{C}} 10.3,10.5,10.8(2 \mathrm{C}, \mathrm{Me}), 37.4,38.1,39.2(\mathrm{CH}), 55.1,55.2,57.1,57.5,57.9(\mathrm{OMe}), 88.9,89.1$, 89.5 (C5), 104.1, 105.3, 106.3, 110.0, 125.9 (aryl C), 128.0, 128.6, 129.0, 129.5, 130.9, 132.0, 132.5 (aryl CH), 135.7, 137.8, 139.1, 140.1, 143.3, 153.1, 154.3 (aryl C). Anal. calcd for $\mathrm{C}_{54} \mathrm{H}_{48} \mathrm{Cl}_{3} \mathrm{~N}_{3} \mathrm{O}_{6} \cdot 2.0 \mathrm{H}_{2} \mathrm{O}: \mathrm{C}, 66.4 ; \mathrm{H}, 5.4 ; \mathrm{N}$, 4.3. Found: $\mathrm{C}, 66.2 ; \mathrm{H}, 5.6 ; \mathrm{N}, 3.5 \%$.

Method b: To a mixture of indole $2(0.25 \mathrm{~g}, 1.31 \mathrm{mmol})$ and 4-chlorobenzaldehyde $(0.18 \mathrm{~g}, 1.30 \mathrm{mmol})$ in chloroform $(25 \mathrm{~mL})$ was added trichloromethylsilane $(0.2 \mathrm{~mL}, 1.7 \mathrm{mmol})$ and the mixture was heated under reflux for $1 \mathrm{~h}$. Cold water was added to the mixture and the chloroform extract washed with $4 \mathrm{M} \mathrm{NaOH}$ until the washings were basic, then dried $\left(\mathrm{MgSO}_{4}\right)$ and concentrated. The residue was chromatographed using dichloromethane/light petroleum (50:50) as eluent to yield compound 35 (110 mg, 27\%).

\section{Acknowledgements}

Financial support from the Australian Research Council is gratefully acknowledged. B.P. also acknowledges receipt of an International Postgraduate Research Scholarship from the Australian Government.

\section{References}

1. Black, D. StC.; Bowyer, M. C.; Kumar, N.; Mitchell, P. S. R. J. Chem. Soc., Chem. Commun. 1993, 819-821. https://doi.org/10.1039/c39930000819

2. Black, D. StC.; Craig, D. C.; Kumar, N. Tetrahedron Lett. 1995, 36, 8075-8078. https://doi.org/10.1016/0040-4039(95)01516-K

3. Black, D. StC.; Craig, D. C.; Kumar, N. Aust. J. Chem. 1996, 49, 311-318. https://doi.org/10.1071/CH9960311

4. Black, D. StC.; Craig, D. C.; Kumar, N.; McConnell, D. B. Tetrahedron Lett. 1996, 37, 241-244. https://doi.org/10.1016/0040-4039(95)02138-8

5. Black, D. StC.; Craig, D. C.; Kumar, N.; McConnell, D. B. Tetrahedron 2000, 56, 8513-8524. https://doi.org/10.1016/S0040-4020(00)00774-2

6. Somphol, K.; Chen, R.; Bhadbhade, M.; Kumar, N; Black, D. StC. Synlett 2013, 24, 24-28.

7. Bonjouklian, R. Synth. Commun. 1985, 15, 711-713. https://doi.org/10.1080/00397918508063862 